DOI 10.4171/JEMS/594

Nir Lev · Joaquim Ortega-Cerdà

\title{
Equidistribution estimates for Fekete points on complex manifolds
}

Received November 14, 2012 and in revised form October 17, 2013

\begin{abstract}
We study the equidistribution of Fekete points in a compact complex manifold. These are extremal point configurations defined through sections of powers of a positive line bundle. Their equidistribution is a known result. The novelty of our approach is that we relate them to the problem of sampling and interpolation on line bundles, which allows us to estimate the equidistribution of the Fekete points quantitatively. In particular we estimate the Kantorovich-Wasserstein distance of the Fekete points to the limiting measure. The sampling and interpolation arrays on line bundles are a subject of independent interest, and we provide necessary density conditions through the classical approach of Landau, that in this context measures the local dimension of the space of sections of the line bundle. We obtain a complete geometric characterization of sampling and interpolation arrays in the case of compact manifolds of dimension one, and we prove that there are no arrays of both sampling and interpolation in the more general setting of semipositive line bundles.
\end{abstract}

Keywords. Beurling-Landau density, Fekete points, holomorphic line bundles

\section{Introduction}

1.1. Let $L$ be a holomorphic line bundle on a compact complex manifold $X$ of dimension $n$. The space of global holomorphic sections of $L$ is denoted by $H^{0}(L)$. If $s_{1}, \ldots, s_{N}$ is a basis for $H^{0}(L)$ and $x_{1}, \ldots, x_{N}$ are $N$ points in $X$, then the Vandermonde-type determinant

$$
\operatorname{det}\left(s_{i}\left(x_{j}\right)\right), \quad 1 \leq i, j \leq N
$$

is a section of the pulled-back line bundle $L^{\otimes N}$ over the manifold $X^{N}$. If $L$ is endowed with a smooth hermitian metric $\phi$, then it also induces a natural metric on $L^{\otimes N}$.

A configuration of $N$ points $x_{1}, \ldots, x_{N}$ in $X$ is called a Fekete configuration for $(L, \phi)$ if it maximizes the pointwise norm $\left|\operatorname{det}\left(s_{i}\left(x_{j}\right)\right)\right|_{\phi}$. It is easy to check that the definition of a Fekete configuration does not depend on the particular choice of the basis $s_{1}, \ldots, s_{N}$ for $H^{0}(L)$. The compactness of $X$ ensures the existence of Fekete configurations (non-unique in general).

N. Lev: Centre de Recerca Matemàtica, Campus de Bellaterra, Edifici C, 08193 Bellaterra (Barcelona), Spain, and Department of Mathematics, Bar-Ilan University, Ramat-Gan 5290002, Israel; e-mail: levnir@math.biu.ac.il

J. Ortega-Cerdà: Dept. Matemàtica Aplicada i Anàlisi and BGSMath, Universitat de Barcelona, Gran Via 585, 08007 Barcelona, Spain; e-mail: jortega@ub.edu

Mathematics Subject Classification (2010): Primary 32U15; Secondary 32L05 
It is interesting to study the distribution of Fekete points with respect to high powers $L^{k}$ of the line bundle $L$, where $L^{k}$ is endowed with the product metric $k \phi$. The model example is the complex projective space $X=\mathbb{C P}^{n}$ with the hyperplane bundle $L=\mathcal{O}(1)$, endowed with the Fubini-Study metric. The $k$-th power of $L$ is denoted $\mathcal{O}(k)$, and the holomorphic sections of $\mathcal{O}(k)$ can be identified with the homogeneous polynomials of degree $k$ in $n+1$ variables. This is in fact the prime example, and it covers in particular the classical theory of weighted orthogonal multivariate polynomials.

For each $k=1,2, \ldots$ let $\mathcal{F}_{k}$ be a Fekete configuration for $\left(L^{k}, k \phi\right)$. The goal is to provide information on the distribution of the Fekete points $\mathcal{F}_{k}$ in geometrical terms of the line bundle $(L, \phi)$, showing that they are "equidistributed" on $X$. We will consider the case when $L$ is an ample line bundle with a smooth positive metric $\phi$. The problem has already been solved by Berman, Boucksom and Witt Nyström [BBWN11] in an even more general context, when $L$ is a big line bundle with an arbitrary continuous metric on a compact subset $K \subset X$. The redeeming feature of our approach is that our new proof provides a quantitative version of the equidistribution.

Theorem 1. If the line bundle $(L, \phi)$ is positive then

$$
\frac{\#\left(\mathcal{F}_{k} \cap B(x, r)\right)}{\# \mathcal{F}_{k}}=\left(1+O\left((r \sqrt{k})^{-1}\right)\right) \frac{\int_{B(x, r)}(i \partial \bar{\partial} \phi)^{n}}{\int_{X}(i \partial \bar{\partial} \phi)^{n}}
$$

for every $r>0$, uniformly in $x \in X$.

Here $\partial \bar{\partial} \phi$ is the curvature form of the metric $\phi$, which is a globally defined $(1,1)$-form on $X$, and $(i \partial \bar{\partial} \phi)^{n}$ is the corresponding volume form on $X$. By $B(x, r)$ we denote the ball of radius $r$ centered at the point $x$ in $X$. To define the balls we endow the manifold $X$ with an arbitrary hermitian metric, and use the associated distance function.

The result shows, in particular, that the weak limit as $k \rightarrow \infty$ of the probability measures

$$
\mu_{k}=\frac{1}{\# \mathcal{F}_{k}} \sum_{\lambda \in \mathcal{F}_{k}} \delta_{\lambda}
$$

is the measure $(i \partial \bar{\partial} \phi)^{n}$ divided by its total mass. This is a special case of the main theorem of [BBWN11] in the setting of positive line bundles.

Theorem 1 provides an even more precise result quantifying the convergence. It measures the discrepancy between the Fekete points and the limit measure. This has been done in the one-dimensional setting (see for instance [RS15, Theorem 3]).

We can also estimate the distance of the Fekete measure $\mu_{k}$ to the limit $v$ in the Kantorovich-Wasserstein metric $W$, which metrizes the weak convergence of measures (see Section 7). This is another measure of how close the Fekete points are to the limit.

Theorem 2. If the line bundle $(L, \phi)$ is positive then

$$
1 / \sqrt{k} \lesssim W\left(\mu_{k}, v\right) \lesssim 1 / \sqrt{k} \quad \text { as } k \rightarrow \infty .
$$

Actually, one can see from the proof of Theorem 2 that the lower bound $1 / \sqrt{k} \lesssim W\left(\mu_{k}, v\right)$ holds for any set of points with the same cardinality as Fekete points. Therefore any 
family of points, no matter how evenly distributed along the manifold with respect to $v$, will converge asymptotically at the same rate as the Fekete points. Thus the Fekete points are in a sense optimally distributed, as expected.

The scheme that we propose to study this problem is along the line of research initiated in [MOC10] where Fekete points are related to another array of points, sampling and interpolation points. This has been pursued further in the one-dimensional setting [AOC12] or even in the real setting of compact Riemannian manifolds [OCP12].

For each $k=1,2, \ldots$ let $\Lambda_{k}$ be a finite set of points in $X$. We assume that $\left\{\Lambda_{k}\right\}$ is a separated array, which means that the distance between any two distinct points in $\Lambda_{k}$ is bounded below by a positive constant times $k^{-1 / 2}$. We say that $\left\{\Lambda_{k}\right\}$ is a sampling array for $(L, \phi)$ if there are constants $0<A, B<\infty$ such that, for each large enough $k$ and any section $s \in H^{0}\left(L^{k}\right)$ we have

$$
A k^{-n} \sum_{\lambda \in \Lambda_{k}}|s(\lambda)|^{2} \leq \int_{X}|s(x)|^{2} \leq B k^{-n} \sum_{\lambda \in \Lambda_{k}}|s(\lambda)|^{2} .
$$

We say that $\left\{\Lambda_{k}\right\}$ is an interpolation array for $(L, \phi)$ if there is a constant $0<C<\infty$ such that, for each large enough $k$ and any set of values $\left\{v_{\lambda}\right\}_{\lambda \in \Lambda_{k}}$, where each $v_{\lambda}$ is an element of the fiber of $\lambda$ in $L^{k}$, there is a section $s \in H^{0}\left(L^{k}\right)$ such that $s(\lambda)=v_{\lambda}\left(\lambda \in \Lambda_{k}\right)$ and

$$
\int_{X}|s(x)|^{2} \leq C k^{-n} \sum_{\lambda \in \Lambda_{k}}\left|v_{\lambda}\right|^{2} .
$$

In order to integrate over $X$ in these definitions we endow $X$ with an arbitrary volume form. It is easy to see that the definitions of sampling and interpolation arrays do not depend on the particular choice of the volume form on $X$.

Our proof of the equidistribution of Fekete points (Theorems 1 and 2 above) is inspired by the work of Nitzan and Olevskii [NO12], who obtained a new proof of a classical result of Landau on the distribution of sampling and interpolation points in the Paley-Wiener space. In some sense, Fekete points are "almost" sampling and interpolation points (see Section 4 below).

1.2. We believe that sampling and interpolation arrays on holomorphic line bundles are a subject of independent interest, so we proceed to a more detailed study of them. We can use Landau's classical technique [Lan67] to get necessary geometric conditions for an array of points to be sampling or interpolation. We could also have used similar techniques to the ones used by Nitzan and Olevskii [NO12] for this purpose. Instead, we have opted for the analysis of Landau's concentration operator, which measures the local dimension of the sections of the line bundle, to obtain necessary density conditions for sampling arrays. This approach was suggested earlier by Berndtsson [Ber03] and Lindholm [Lin01] in the context of holomorphic line bundles. ratio

Let $v_{\Lambda}^{-}(R)$ (respectively $v_{\Lambda}^{+}(R)$ ) denote the infimum (respectively supremum) of the

$$
\frac{k^{-n} \#\left(\Lambda_{k} \cap B(x, r)\right)}{\int_{B(x, r)}(i \partial \bar{\partial} \phi)^{n}}
$$


over all $x \in X$ and all $k, r$ such that $R / \sqrt{k} \leq r \leq \operatorname{diam}(X)$. As before, to define the balls $B(x, r)$ we have fixed an arbitrary hermitian metric on the manifold $X$.

Theorem 3. Let the line bundle $(L, \phi)$ be positive, and $\Lambda=\left\{\Lambda_{k}\right\}$ be a separated array.

(i) If $\Lambda$ is a sampling array then

$$
v_{\Lambda}^{-}(R)>\frac{1}{\pi^{n} n !}-O\left(R^{-1}\right), \quad R \rightarrow \infty .
$$

(ii) If $\Lambda$ is an interpolation array then

$$
v_{\Lambda}^{+}(R)<\frac{1}{\pi^{n} n !}+O\left(R^{-1}\right), \quad R \rightarrow \infty .
$$

This result yields necessary conditions in terms of the lower and upper Beurling-Landau densities, defined by

$$
D^{-}(\Lambda)=\liminf _{R \rightarrow \infty} v_{\Lambda}^{-}(R) \text { and } D^{+}(\Lambda)=\limsup _{R \rightarrow \infty} v_{\Lambda}^{+}(R) .
$$

Corollary 1. Let the line bundle $(L, \phi)$ be positive and $\Lambda=\left\{\Lambda_{k}\right\}$ be a separated array. If $\Lambda$ is a sampling array then

$$
D^{-}(\Lambda) \geq \frac{1}{\pi^{n} n !}
$$

while if $\Lambda$ is an interpolation array then

$$
D^{+}(\Lambda) \leq \frac{1}{\pi^{n} n !}
$$

When the complex manifold $X$ is one-dimensional, i.e. we are dealing with a compact Riemann surface, we have a more precise result. In this case there is a complete geometric characterization of sampling and interpolation arrays in terms of the above densities.

Theorem 4. Let $(L, \phi)$ be a positive line bundle over a compact Riemann surface $X$, and let $\Lambda=\left\{\Lambda_{k}\right\}$ be a separated array. Then $\Lambda$ is a sampling array if and only if

$$
D^{-}(\Lambda)>1 / \pi
$$

while it is an interpolation array if and only if

$$
D^{+}(\Lambda)<1 / \pi
$$

We remark that the assumption that $\left\{\Lambda_{k}\right\}$ is separated is not essential, and similar results hold in the general case. This can be done with standard techniques (see e.g. [Mar07]), so we will not go into details.

1.3. As pointed out in $[\mathrm{MOC} 10]$, Fekete points provide a construction of an "almost" sampling and interpolation array, with the critical density. In particular this shows that the density threshold in Corollary 1 is sharp (see Corollary 3 in Section 7). 
In this context a natural question is whether the array of Fekete points, or possibly some other array of points, is simultaneously sampling and interpolation for $(L, \phi)$. When the manifold $X$ is one-dimensional, this question is settled in the negative by Theorem 4 above. For $n>1$ we do not have strict density conditions, and Corollary 1 does not exclude the existence of simultaneously sampling and interpolation arrays. Nevertheless, we will show that such arrays do not exist, even in the more general setting when the metric $\phi$ is semipositive and has at least one point with a strictly positive curvature.

Theorem 5. Let $L$ be a holomorphic line bundle over a compact projective manifold $X$, and $\phi$ be a semipositive smooth hermitian metric on L. If there is a point in $X$ where $\phi$ has a strictly positive curvature, then there are no arrays which are simultaneously sampling and interpolation for $(L, \phi)$.

Here we need to assume that the manifold $X$ is projective. When the line bundle is positive this is automatically the case, according to the Kodaira embedding theorem [Kod54].

The non-existence of simultaneously sampling and interpolation sequences is a recent result in the classical Bargmann-Fock space [AFK14, GM13]. To prove Theorem 5 we use the fact that near a point of positive curvature, sections of high powers of a line bundle closely resemble functions in the Bargmann-Fock space. Also our proof of Theorem 4 is guided by the same principle.

1.4. The plan of the paper is the following. In Sections 2 and 3 we provide the basic properties of Fekete points, and of the Hilbert space of holomorphic sections that will be the main tool to study them. In Section 4 we introduce sampling and interpolation arrays and discuss their relationship with Fekete points. In Section 5 we study Landau's concentration operator, which will allow us to measure the local dimension of the space of sections essentially concentrated in a given ball, and use this local dimension to estimate the number of points in an interpolation or sampling array. In Section 6 we estimate the density of interpolation and sampling arrays in terms of the volume form associated to the curvature of the line bundle. In Section 7 we give an estimate from above and below on the number of Fekete points that lie in a given ball. We also provide the upper and lower bounds for the Kantorovich-Wasserstein distance between the Fekete measure (1) and its limiting measure.

Next we proceed to a more detailed study of sampling and interpolation arrays. In Section 8 we prove that in a big line bundle with a semipositive metric, whenever there is a point of positive curvature there are no arrays that are simultaneously sampling and interpolation. Finally in Section 9 we obtain a geometric characterization of sampling and interpolation arrays for positive line bundles over compact manifolds of dimension one.

\section{Preliminaries}

In this section we recall some basic properties of holomorphic line bundles over complex manifolds. For these and other elementary facts on this subject, stated below without proofs, the reader may consult [Ber10]. 
2.1. Line bundles. Below, $X$ will be a compact complex manifold of dimension $n$, endowed with a smooth hermitian metric $\omega$. The metric $\omega$ induces a distance function $d(x, y)$ on $X$, which will be used to define the balls $B(x, r)=\{y \in X: d(x, y)<r\}$. The hermitian metric $\omega$ also induces a volume form $V$ on $X$, which will be used to integrate over $X$. We emphasize that the choice of the metric $\omega$ is arbitrary, and the results will not depend on the particular choice made.

By $L$ we denote a holomorphic line bundle over the manifold $X$. We assume that $L$ is endowed with a smooth hermitian metric $\phi$, which is a smoothly varying norm on each fiber. It has to be understood as a collection of functions $\phi_{i}$ defined on trivializing open sets $U_{i}$ which cover $X$, and satisfying the compatibility conditions

$$
\phi_{i}-\phi_{j}=\log \left|g_{i j}\right|^{2},
$$

where $g_{i j}$ are the transition functions of the line bundle $L$ on $U_{i} \cap U_{j}$. If $s$ is a section of $L$ represented by a collection of local functions $s_{i}$ such that $s_{i}=g_{i j} s_{j}$, then

$$
|s(x)|^{2}=\left|s_{i}(x)\right|^{2} e^{-\phi_{i}(x)} .
$$

We also have an associated scalar product, defined in a similar way by

$$
\langle u(x), v(x)\rangle=u_{i}(x) \overline{v_{i}(x)} e^{-\phi_{i}(x)} .
$$

If $\phi$ is the hermitian metric on $L$, then $\partial \bar{\partial} \phi$ is a globally defined $(1,1)$-form on $X$, which is called the curvature form of the metric $\phi$. The line bundle $L$ with metric $\phi$ is called positive if $i \partial \bar{\partial} \phi$ is a positive form. Equivalently, $L$ with metric $\phi$ is positive if the representative of $\phi$ in any local trivialization is a strictly plurisubharmonic function. We remark that when $\phi$ is positive, the curvature form $\partial \bar{\partial} \phi$ may be used to define a natural metric on $X$, which in turn induces a distance function and a volume form on $X$. However, we find it convenient to work with an arbitrary metric $\omega$, which is not necessarily related to the curvature form.

The notation $\lesssim$ will be understood to involve an implicit multiplicative constant which may depend only on the hermitian manifold $(X, \omega)$ and the hermitian line bundle $(L, \phi)$.

The space of global holomorphic sections of $L$ will be denoted $H^{0}(L)$. This is a finite-dimensional space satisfying

$$
\operatorname{dim} H^{0}\left(L^{k}\right) \lesssim k^{n}
$$

While this estimate holds for an arbitrary line bundle on a compact manifold, when the line bundle $L$ is big there is also a similar estimate from below, i.e.

$$
k^{n} \lesssim \operatorname{dim} H^{0}\left(L^{k}\right) \lesssim k^{n}
$$

In particular this holds whenever $L$ is positive.

If $L$ is a line bundle over $X$ and $M$ is a line bundle over $Y$, we denote by $L \otimes M$ the line bundle over the product manifold $X \times Y$ defined as $L \otimes M=\pi_{X}^{*}(L) \otimes \pi_{Y}^{*}(M)$, where $\pi_{X}$ and $\pi_{Y}$ are the projections of $X \times Y$ onto $X$ and $Y$ respectively. 
2.2. Bergman kernel. The space $H^{0}(L)$ admits a Hilbert space structure when endowed with the scalar product

$$
\langle u, v\rangle=\int_{X}\langle u(x), v(x)\rangle, \quad u, v \in H^{0}(L),
$$

where integration is with respect to the volume form $V$.

The Bergman kernel $\Pi(x, y)$ associated to this space is a section of the line bundle $L \otimes \bar{L}$ over $X \times X$, defined by

$$
\Pi(x, y)=\sum_{j=1}^{N} s_{j}(x) \otimes \overline{s_{j}(y)},
$$

where $s_{1}, \ldots, s_{N}$ is an orthonormal basis of $H^{0}(L)$. It is easy to check that this definition does not depend on the particular choice of the orthonormal basis $s_{1}, \ldots, s_{N}$. The Bergman kernel $\Pi(x, y)$ is in a sense the reproducing kernel for the space $H^{0}(L)$, satisfying the reproducing formula

$$
s(x)=\int_{X}\langle s(y), \Pi(x, y)\rangle d V(y)
$$

for $s \in H^{0}(L)$. The pointwise norm of the Bergman kernel is symmetric,

$$
|\Pi(x, y)|=|\Pi(y, x)| .
$$

The function $|\Pi(x, x)|$ is called the Bergman function of $H^{0}(L)$. It can be expressed as

$$
|\Pi(x, x)|=\sum_{j=1}^{N}\left|s_{j}(x)\right|^{2},
$$

and it satisfies

$$
|\Pi(x, x)|=\int_{X}|\Pi(x, y)|^{2} d V(y) .
$$

Lemma 1. Let $y \in X$. There is a section $\Phi_{y} \in H^{0}(L)$ such that

$$
\left|\Phi_{y}(x)\right|=|\Pi(x, y)|, \quad x \in X .
$$

Proof. Let $s_{1}, \ldots, s_{N}$ be an orthonormal basis for $H^{0}(L)$. Fix a frame $e(x)$ in a neighborhood $U$ of $y$. Then in this neighborhood each $s_{j}$ is represented by a holomorphic function $f_{j}$ such that $s_{j}(x)=f_{j}(x) e(x)$. Define

$$
\Phi_{y}(x):=|e(y)| \sum_{j=1}^{n} \overline{f_{j}(y)} s_{j}(x) .
$$

Then $\Phi_{y}$ is a holomorphic section of $L$, and we have

$$
\left|\Phi_{y}(x)\right|=\left|\left(\sum_{j=1}^{N} \overline{f_{j}(y)} s_{j}(x)\right) \otimes \overline{e(y)}\right|=\left|\sum_{j=1}^{N} s_{j}(x) \otimes \overline{s_{j}(y)}\right|=|\Pi(x, y)| .
$$


We denote by $\Pi_{k}(x, y)$ the Bergman kernel for the $k$-th power $L^{k}$ of the line bundle $L$ (where $L^{k}$ is endowed with the product metric $k \phi$ ). The behavior of $\Pi_{k}(x, y)$ as $k \rightarrow \infty$ is of special importance. In the case when the line bundle $(L, \phi)$ is positive, it is known (see e.g. [Ber03, Lin01]) that

$$
k^{n} \lesssim\left|\Pi_{k}(x, x)\right| \lesssim k^{n}
$$

and

$$
\left|\Pi_{k}(x, y)\right| \lesssim k^{n} \exp (-c \sqrt{k} d(x, y)),
$$

where $c=c(X, \omega, L, \phi)$ is an appropriate positive constant.

2.3. Sub-mean value property. Let $s \in H^{0}\left(L^{k}\right)$. If $z \in X$ and $0<\delta<1$, then

$$
|s(z)|^{p} \lesssim p\left(\frac{\delta}{\sqrt{k}}\right)^{-2 n} \int_{B(z, \delta / \sqrt{k})}|s(x)|^{p} \quad(1 \leq p<\infty) .
$$

where $\lesssim_{p}$ indicates that the implicit constant may also depend on $p$. This can be deduced easily from the compactness of $X$ and the corresponding fact in $\mathbb{C}^{n}$, which may be found for example in [Lin01, Lemma 7].

As a consequence we have the following Plancherel-Pólya type inequality:

Lemma 2. Let $\left\{x_{j}\right\}$ be points in $X$ such that $d\left(x_{i}, x_{j}\right) \geq \delta / \sqrt{k}$ for some $0<\delta<1$. Then

$$
k^{-n} \sum_{j}\left|s\left(x_{j}\right)\right|^{p} \lesssim \delta^{-2 n} \int_{X}|s(x)|^{p} \quad(1 \leq p<\infty)
$$

for any $s \in H^{0}\left(L^{k}\right)$

\section{Fekete points and their properties}

3.1. Let $N=\operatorname{dim} H^{0}(L)$, and $s_{1}, \ldots, s_{N}$ be a basis for $H^{0}(L)$. A configuration of $N$ points $x_{1}, \ldots, x_{N}$ in $X$ is called a Fekete configuration if it maximizes the pointwise norm of the Vandermonde-type determinant

$$
\operatorname{det}\left(s_{i}\left(x_{j}\right)\right), \quad 1 \leq i, j \leq N
$$

which is a holomorphic section to the line bundle $L^{\otimes N}$ over the manifold $X^{N}$ (endowed with the metric inherited from $L$ ).

If $e_{j}(x)$ is a frame in a neighborhood $U_{j}$ of the point $x_{j}$, then the sections $s_{i}(x)$ are represented on each $U_{j}$ by scalar functions $f_{i j}$ such that $s_{i}(x)=f_{i j}(x) e_{j}(x)$. Similarly, the metric $\phi$ is represented on $U_{j}$ by a smooth real-valued function $\phi_{j}$ such that $\left|s_{i}(x)\right|^{2}=$ $\left|f_{i j}(x)\right|^{2} e^{-\phi_{j}(x)}$. A Fekete configuration thus maximizes the quantity

$$
e^{-\phi_{1}\left(x_{1}\right)} \cdots e^{-\phi_{N}\left(x_{N}\right)}\left|\operatorname{det}\left(f_{i j}\left(x_{j}\right)\right)\right|^{2} .
$$


By the compactness of $X$, Fekete configurations exist, but in general need not be unique. One may check that the norm $\left|\operatorname{det}\left(s_{i}\left(x_{j}\right)\right)\right|_{\phi}$ at a Fekete configuration $x_{1}, \ldots, x_{N}$ is always non-zero. It is also easy to check that the definition of a Fekete configuration does not depend on the particular choice of the basis $s_{1}, \ldots, s_{N}$ of $H^{0}(L)$.

The function (11) is a Vandermonde-type determinant that vanishes when two points are equal. It is exactly the familiar Vandermonde determinant in the special case when the sections $s_{i}$ are the monomials in dimension one, and the weight $\phi$ is constant. This suggests what is actually happening - the Fekete points repel each other and tend to be in a sense "maximally spread".

3.2. The main property of the Fekete points $x_{1}, \ldots, x_{N}$ that will be used is the existence of "Lagrange sections" with a uniformly bounded norm. Namely, there are sections $\ell_{1}, \ldots, \ell_{N}$ in $H^{0}(L)$ such that

$$
\left|\ell_{j}\left(x_{i}\right)\right|=\delta_{i j}, \quad 1 \leq i, j \leq N,
$$

and moreover

$$
\sup _{x \in X}\left|\ell_{j}(x)\right|=1, \quad 1 \leq j \leq N
$$

To construct such sections we denote by $M$ the matrix $\left(e^{-\frac{1}{2} \phi_{j}\left(x_{j}\right)} f_{i j}\left(x_{j}\right)\right)$, and define

$$
\ell_{j}(x):=\frac{1}{\operatorname{det}(M)} \sum_{i=1}^{N}(-1)^{i+j} M_{i j} s_{i}(x)
$$

where $M_{i j}$ is the determinant of the submatrix obtained from $M$ by removing the $i$-th row and $j$-th column. Clearly $\ell_{j} \in H^{0}(L)$, and it is not difficult to check that conditions (12) and (13) above hold, where (13) is a consequence of the extremal property of the Fekete configuration $x_{1}, \ldots, x_{N}$.

We also observe that the system $\left\{\ell_{j}(x)\right\}$ forms a basis of $H^{0}(L)$. Indeed, the condition (12) implies that the $\ell_{j}(x)$ are linearly independent, and since they form a system with $N$ elements, $N=\operatorname{dim} H^{0}(L)$, they span the whole $H^{0}(L)$. An element $s \in H^{0}(L)$ thus has a unique expansion

$$
s(x)=\sum_{j=1}^{N} c_{j} \ell_{j}(x),
$$

and the coefficients $c_{j}$ are given by

$$
c_{j}=\left\langle s\left(x_{j}\right), \ell_{j}\left(x_{j}\right)\right\rangle, \quad 1 \leq j \leq N
$$

which again follows from (12). 
3.3. One consequence of the construction above is that Fekete points form a separated array.

Lemma 3. Let $\mathcal{F}_{k}$ be a Fekete configuration for $\left(L^{k}, k \phi\right)$. Then

$$
d(x, y) \gtrsim 1 / \sqrt{k}, \quad x, y \in \mathcal{F}_{k}, x \neq y .
$$

Proof. Indeed, if this is not the case, then there are points $x_{k}, y_{k} \in \mathcal{F}_{k}$ with $\sqrt{k} d\left(x_{k}, y_{k}\right)$ $\rightarrow 0$ but $x_{k} \neq y_{k}$ for infinitely many $k$ 's. By compactness we may assume that $x_{k}, y_{k}$ converge to some point $x \in X$. We choose local coordinates $z$ in a neighborhood of $x$, and a local trivialization of the line bundle $L$ in this neighborhood. The metric on $L$ is represented by a smooth function $\phi(z)$, and the metric on $L^{k}$ is given by $k \phi(z)$.

For each $k$, we have a "Lagrange section" vanishing at $x_{k}$ and having norm one at $y_{k}$. Let it be given by a holomorphic function $f_{k}(z)$ in the local trivialization. Thus

$$
\left|f_{k}(z)\right|^{2} e^{-k \phi(z)}= \begin{cases}0, & z=z\left(x_{k}\right), \\ 1, & z=z\left(y_{k}\right),\end{cases}
$$

and $\left|f_{k}(z)\right|^{2} e^{-k \phi(z)} \leq 1$ for all other $z$

On the other hand, the distance function $d$ is equivalent to the Euclidean distance with respect to the local coordinates. Hence,

$$
\sqrt{k}\left|z\left(x_{k}\right)-z\left(y_{k}\right)\right| \rightarrow 0 \quad(k \rightarrow \infty) .
$$

This implies that the norm of the gradient of $|f|^{2} e^{-k \phi}$ must be, at some point $z_{k}$, larger than $\sqrt{k}$ times a quality tending to infinity. However, Lemma 4 below shows that this is impossible, and this contradiction concludes the proof of Lemma 3.

Lemma 4. Let $\phi(z)$ be a smooth, real-valued function in a neighborhood of the point $w=\left(w_{1}, \ldots, w_{n}\right) \in \mathbb{C}^{n}$. Then there are constants $C$ and $k_{0}$ such that the following holds. Let $k \geq k_{0}$, and $f(z)$ be a holomorphic function in a neighborhood of the compact set

$$
U_{k}(w)=\left\{z \in \mathbb{C}^{n}:\left|z_{j}-w_{j}\right| \leq 1 / \sqrt{k}(j=1, \ldots, n)\right\} .
$$

Then for each $1 \leq j \leq n$ we have

$$
\left|\frac{\partial}{\partial z_{j}}\left[|f|^{2} e^{-k \phi}\right](w)\right| \leq C \sqrt{k} \sup _{U_{k}(w)}|f|^{2} e^{-k \phi} .
$$

This is proved in dimension one in [AOC12, Lemma 19(b)]. The multi-dimensional version above can be proved in a similar way.

If the line bundle $(L, \phi)$ is positive, the separation condition (14) of the Fekete array is sharp in a sense. The following is true.

Lemma 5. If $(L, \phi)$ is positive then there is $R>0$, not depending on $k$, with the following property: if $\mathcal{F}_{k}$ is a Fekete configuration for $\left(L^{k}, k \phi\right)$, then any ball $B(x, R / \sqrt{k})$, $x \in X$, contains at least one point of $\mathcal{F}_{k}$.

This result may be deduced from Theorem 3 and Lemma 6 below. However, as it will not be used later on, we do not present the details of the proof. We merely state it to show that the Fekete points $\mathcal{F}_{k}$ are roughly at a distance $1 / \sqrt{k}$ from each other. 


\section{Sampling and interpolation arrays}

4.1. In this section we relate Fekete arrays to sampling and interpolation arrays. We will show that if the line bundle $(L, \phi)$ is positive, then by a "small perturbation" of a Fekete array one obtains a sampling or interpolation array for $(L, \phi)$.

Definition 1. Let $k$ be a positive integer, and $\Lambda_{k}$ be a finite set of points in $X$. We say that $\Lambda_{k}$ is a sampling set at level $k$ with sampling constants $A, B$ if

$$
A k^{-n} \sum_{\lambda \in \Lambda_{k}}|s(\lambda)|^{2} \leq \int_{X}|s(x)|^{2} \leq B k^{-n} \sum_{\lambda \in \Lambda_{k}}|s(\lambda)|^{2}
$$

for any section $s \in H^{0}\left(L^{k}\right)$. We say that $\Lambda_{k}$ is an interpolation set at level $k$ with interpolation constant $C$ if for any set $\left\{v_{\lambda}\right\}_{\lambda \in \Lambda_{k}}$, where each $v_{\lambda}$ is an element of the fiber of $\lambda$ in $L^{k}$, there is a section $s \in H^{0}\left(L^{k}\right)$ such that $s(\lambda)=v_{\lambda}\left(\lambda \in \Lambda_{k}\right)$ and

$$
\int_{X}|s(x)|^{2} \leq C k^{-n} \sum_{\lambda \in \Lambda_{k}}\left|v_{\lambda}\right|^{2}
$$

Definition 2. Let $\Lambda=\left\{\Lambda_{k}\right\}$ be an array of points, i.e. a sequence of finite sets $\Lambda_{k}$ in $X$. We call $\Lambda$ a sampling array if there are $k_{0}$ and positive constants $A, B$, not depending on $k$, such that $\Lambda_{k}$ is a sampling set at each level $k \geq k_{0}$ with sampling constants $A, B$. Analogously, $\Lambda$ is an interpolation array if there are $k_{0}$ and a positive constant $C$, not depending on $k$, such that $\Lambda_{k}$ is an interpolation set at each level $k \geq k_{0}$ with interpolation constant $C$.

Lemma 6. Suppose that $(L, \phi)$ is positive. Let $k$ be a positive integer, and $\varepsilon$ be a number satisfying $1 / k \lesssim \varepsilon \lesssim 1$. If we define

$$
\Lambda_{k}:=\mathcal{F}_{(1+\varepsilon) k}
$$

then $\Lambda_{k}$ is a sampling set at level $k$ with sampling constants $A, B$ such that $1 \lesssim A<$ $B \lesssim \varepsilon^{-2 n}$. On the other hand, the set

$$
\Lambda_{k}:=\mathcal{F}_{(1-\varepsilon) k}
$$

is an interpolation set at level $k$ with interpolation constant $C \lesssim \varepsilon^{-2 n}$.

We must provide a clarification concerning the statement of the theorem: we have written $\mathcal{F}_{(1 \pm \varepsilon) k}$ as if the numbers $(1 \pm \varepsilon) k$ were integers. In practice, the reader should replace these numbers by an integer approximation. The same is true in other parts of the paper.

It follows from Lemma 6 that by a "small perturbation" of a Fekete array one obtains a sampling or interpolation array for $(L, \phi)$.

Corollary 2. Let $(L, \phi)$ be positive, and $\varepsilon>0$ be fixed. Then

(i) $\left\{\mathcal{F}_{(1+\varepsilon) k}\right\}$ is a sampling array for $(L, \phi)$;

(ii) $\left\{\mathcal{F}_{(1-\varepsilon) k}\right\}$ is an interpolation array for $(L, \phi)$.

The rest of this section is devoted to the proof of Lemma 6. 
4.2. We start with the interpolation part of Lemma 6 . We fix $k$ and $\varepsilon$ satisfying $1 / k \lesssim$ $\varepsilon \lesssim 1$ and define $\Lambda_{k}=\mathcal{F}_{(1-\varepsilon) k}$. We will prove that $\Lambda_{k}$ is an interpolation set at level $k$ with interpolation constant $C \lesssim \varepsilon^{-2 n}$.

Denote by $\left\{x_{j}\right\}$ the elements of the finite set $\Lambda_{k}$. Since the points $\left\{x_{j}\right\}$ form a Fekete configuration for the line bundle $L^{(1-\varepsilon) k}$, they have associated Lagrange sections $\ell_{j}$ (see Section 3). The sections $\ell_{j}$ are suitable for solving the interpolation problem with nodes $x_{j}$, but we also need an estimate for the $L^{2}$ norm of the solution. For this reason we need to improve the localization of $\ell_{j}$ around the point $x_{j}$. We therefore define the auxiliary sections

$$
Q_{j}(x):=\ell_{j}(x) \otimes\left[\frac{\Phi_{x_{j}}^{(\varepsilon / 2) k}(x)}{\left|\Pi_{(\varepsilon / 2) k}\left(x_{j}, x_{j}\right)\right|}\right]^{2} \in H^{0}\left(L^{k}\right),
$$

where $\Phi_{y}^{(\varepsilon / 2) k}$ denotes a holomorphic section to $L^{(\varepsilon / 2) k}$ such that

$$
\left|\Phi_{y}^{(\varepsilon / 2) k}(x)\right|=\left|\Pi_{(\varepsilon / 2) k}(x, y)\right|, \quad x \in X .
$$

The existence of such a section is guaranteed by Lemma 1.

We have thus constructed sections $Q_{j}$ in $H^{0}\left(L^{k}\right)$ which are associated to the points $\left\{x_{j}\right\}$. Similar to the Lagrange sections, the sections $Q_{j}$ satisfy

$$
\left|Q_{j}\left(x_{i}\right)\right|=\delta_{i j},
$$

as follows from (7) and (12). We will also need the additional estimates

$$
\begin{aligned}
& \sup _{j} \int_{X}\left|Q_{j}(x)\right| \lesssim(\varepsilon k)^{-n}, \\
& \sup _{x \in X} \sum_{j}\left|Q_{j}(x)\right| \lesssim \varepsilon^{-n},
\end{aligned}
$$

which will be proved now. The inequality (19) follows directly from (7), (8) and (13). To prove (20) we recall that Fekete points are separated (Lemma 3), and hence

$$
d\left(x_{i}, x_{j}\right) \gtrsim \frac{1}{\sqrt{(1-\varepsilon) k}} \gtrsim \frac{\delta}{\sqrt{(\varepsilon / 2) k}}
$$

with $\delta=\sqrt{\varepsilon}$. Thus an application of the Plancherel-Pólya inequality (Lemma 2) to the section $\Phi_{x}^{(\varepsilon / 2) k}$ and to the set $\left\{x_{j}\right\}$ yields

$$
\sum_{j}\left|Q_{j}(x)\right| \lesssim(\varepsilon k)^{-2 n} \sum_{j}\left|\Phi_{x}^{(\varepsilon / 2) k}\left(x_{j}\right)\right|^{2} \lesssim \varepsilon^{-2 n} k^{-n} \int_{X}\left|\Phi_{x}^{(\varepsilon / 2) k}\right|^{2} \lesssim \varepsilon^{-n},
$$

where we have used (5), (7), (8) and (17).

We are now ready to solve the interpolation problem with estimate. Suppose that we are given a set $\left\{v_{j}\right\}$, where each $v_{j}$ is an element of the fiber of $x_{j}$ in $L^{k}$. We will 
construct a solution $Q(x)$ to the interpolation problem, i.e. a section $Q \in H^{0}\left(L^{k}\right)$ such that $Q\left(x_{j}\right)=v_{j}$ for all $j$. The solution is defined as a linear combination of the $Q_{j}$,

$$
Q(x)=\sum_{j} c_{j} Q_{j}(x)
$$

with the coefficients $c_{j}$ given by $c_{j}=\left\langle v_{j}, Q_{j}\left(x_{j}\right)\right\rangle$. This choice of the coefficients and the property (18) imply that $Q(x)$ is indeed a solution to the interpolation problem.

It remains to show that the solution $Q(x)$ is bounded in $L^{2}$ with

$$
\int_{X}|Q(x)|^{2} \lesssim \varepsilon^{-2 n} k^{-n} \sum_{j}\left|v_{j}\right|^{2}
$$

Indeed, by the Cauchy-Schwarz inequality and (20) we have

$$
|Q(x)|^{2} \leq\left(\sum_{j}\left|c_{j}\right|^{2}\left|Q_{j}(x)\right|\right)\left(\sum_{j}\left|Q_{j}(x)\right|\right) \lesssim \varepsilon^{-n} \sum_{j}\left|c_{j}\right|^{2}\left|Q_{j}(x)\right| .
$$

Integrating over $X$ and using (19) yields

$$
\int_{X}|Q(x)|^{2} \lesssim \varepsilon^{-n} \sum_{j}\left|c_{j}\right|^{2} \int_{X}\left|Q_{j}(x)\right| \lesssim \varepsilon^{-2 n} k^{-n} \sum_{j}\left|c_{j}\right|^{2},
$$

and since $\left|c_{j}\right|=\left|v_{j}\right|$ this gives (21).

This completes the proof of the interpolation part of Lemma 6 .

4.3. We turn to the proof of the sampling part of Lemma 6. In this case we are dealing with the set $\Lambda_{k}=\mathcal{F}_{(1+\varepsilon) k}$, and must prove that it is a sampling set at level $k$ with sampling constants $A, B$ such that $1 \lesssim A<B \lesssim \varepsilon^{-2 n}$.

Again we denote by $\left\{x_{j}\right\}$ the elements of $\Lambda_{k}$. We will prove the sampling inequality

$$
k^{-n} \sum_{j}\left|s\left(x_{j}\right)\right|^{2} \lesssim \int_{X}|s(x)|^{2} \lesssim \varepsilon^{-2 n} k^{-n} \sum_{j}\left|s\left(x_{j}\right)\right|^{2}
$$

for any section $s \in H^{0}\left(L^{k}\right)$. The left hand inequality of (23) is a consequence of the Plancherel-Pólya inequality (Lemma 2 ) and the separation condition

$$
d\left(x_{i}, x_{j}\right) \gtrsim 1 / \sqrt{(1+\varepsilon) k} \gtrsim 1 / \sqrt{k}
$$

ensured by Lemma 3.

The proof of the right hand inequality of (23) is similar to the interpolation part. Fix $x \in X$ and define

$$
P_{x}(y):=s(y) \otimes\left[\frac{\Phi_{x}^{(\varepsilon / 2) k}(y)}{\left|\Pi_{(\varepsilon / 2) k}(x, x)\right|}\right]^{2} \in H^{0}\left(L^{(1+\varepsilon) k}\right) .
$$


The space $H^{0}\left(L^{(1+\varepsilon) k}\right)$ has a basis of Lagrange sections $\ell_{j}$ associated to the Fekete points $\left\{x_{j}\right\}$, so we may expand $P_{x}$ in terms of this basis. We get

$$
P_{x}(y)=\sum_{j}\left\langle P_{x}\left(x_{j}\right), \ell_{j}\left(x_{j}\right)\right\rangle \ell_{j}(y) .
$$

In particular, if $y=x$ this implies

$$
|s(x)|=\left|P_{x}(x)\right| \leq \sum_{j}\left|P_{x}\left(x_{j}\right)\right|=\sum_{j}\left|s\left(x_{j}\right)\right|\left|Q_{j}(x)\right|,
$$

where we now define

$$
Q_{j}(x):=\left[\frac{\Phi_{x_{j}}^{(\varepsilon / 2) k}(x)}{\left|\Pi_{(\varepsilon / 2) k}(x, x)\right|}\right]^{2} .
$$

The estimates (19), (20) are valid in this case as well, and can be proved in the same way. We may therefore continue as in (22). We obtain

$$
|s(x)|^{2} \leq\left(\sum_{j}\left|s\left(x_{j}\right)\right|^{2}\left|Q_{j}(x)\right|\right)\left(\sum_{j}\left|Q_{j}(x)\right|\right) \lesssim \varepsilon^{-n} \sum_{j}\left|s\left(x_{j}\right)\right|^{2}\left|Q_{j}(x)\right|,
$$

and integrating over $X$ yields the right hand inequality of (23).

We have thus also proved the sampling part of Lemma 6 , so the lemma is completely proved.

Remark 1. In the proof of Lemma 6 we have not used any off-diagonal estimate such as (9) for the Bergman kernel, but only the asymptotic estimate (8) on the diagonal combined with the $L^{2}$ equality (7) (this is in contrast to [AOC12], for example).

\section{Landau's inequalities}

5.1. In this section we use Landau's method [Lan67] to obtain estimates for the number of points of a separated sampling or interpolation array in a ball.

Let us say that a finite set of points $\Lambda_{k}$ is $\delta$-separated at level $k$ if

$$
d(x, y) \geq \delta / \sqrt{k}, \quad x, y \in \Lambda_{k}, x \neq y .
$$

Our goal is to prove the following two statements.

Lemma 7. Let $\Lambda_{k}$ be a $\delta$-separated sampling set at level $k$ with sampling constants $A, B$. Then for any $z \in X$ and $r>0$,

$$
\#\left(\Lambda_{k} \cap B(z,(r+\delta) / \sqrt{k})\right) \geq \int_{\Omega}\left|\Pi_{k}(x, x)\right|-M \iint_{\Omega \times \Omega^{c}}\left|\Pi_{k}(x, y)\right|^{2},
$$

where $\Omega=B(z, r / \sqrt{k})$, and the constant $M$ is bounded by the sampling constant $B$ times a constant which may depend on $\delta$ but does not depend on $k, z, r$. 
Lemma 8. Similarly, if $\Lambda_{k}$ is a $\delta$-separated interpolation set at level $k$ with interpolation constant $C$, then for any $z \in X$ and $r>0$,

$$
\#\left(\Lambda_{k} \cap B(z,(r-\delta) / \sqrt{k})\right) \leq \int_{\Omega}\left|\Pi_{k}(x, x)\right|+M \iint_{\Omega \times \Omega^{c}}\left|\Pi_{k}(x, y)\right|^{2},
$$

where again $\Omega=B(z, r / \sqrt{k})$, and the constant $M$ is bounded by the interpolation constant $C$ times a constant which may depend on $\delta$ but does not depend on $k, z, r$.

5.2. Let $\Omega$ be a measurable subset of $X$. We denote by $T_{\Omega}$ the linear operator on $H^{0}(L)$ defined by

$$
T_{\Omega}(s)=P\left(s \cdot \mathbb{1}_{\Omega}\right), \quad s \in H^{0}(L),
$$

where $P$ denotes the orthogonal projection from the Hilbert space of all $L^{2}$ sections onto its finite-dimensional subspace $H^{0}(L)$. It is easy to see that

$$
\left\langle T_{\Omega} s, s\right\rangle=\int_{\Omega}|s|^{2}, \quad s \in H^{0}(L),
$$

hence $T_{\Omega}$ is self-adjoint, non-negative and $\left\|T_{\Omega}\right\| \leq 1$. We may therefore find an orthonormal basis $\left\{s_{j}\right\}$ of $H^{0}(L)$ consisting of eigensections,

$$
T_{\Omega}\left(s_{j}\right)=\lambda_{j}(\Omega) s_{j} .
$$

The eigenvalues $\lambda_{j}(\Omega)$ lie between 0 and 1 , and we arrange them in non-increasing order,

$$
\lambda_{1}(\Omega) \geq \lambda_{2}(\Omega) \geq \cdots \geq 0 .
$$

By using (6) with the basis $\left\{s_{j}\right\}$ of eigensections we can compute the trace of $T_{\Omega}$,

$$
\sum_{j \geq 1} \lambda_{j}(\Omega)=\sum_{j \geq 1}\left\langle T_{\Omega} s_{j}, s_{j}\right\rangle=\sum_{j \geq 1} \int_{\Omega}\left|s_{j}(x)\right|^{2}=\int_{\Omega}|\Pi(x, x)| .
$$

Similarly, (4) allows us to compute the Hilbert-Schmidt norm of $T_{\Omega}$ (the trace of $T_{\Omega}^{2}$ ) in terms of the Bergman kernel. Indeed,

$$
|\Pi(x, y)|^{2}=\sum_{j \geq 1} \sum_{k \geq 1}\left\langle s_{j}(x), s_{k}(x)\right\rangle \overline{\left\langle s_{j}(y), s_{k}(y)\right\rangle},
$$

hence integrating over $\Omega \times \Omega$ gives

$$
\sum_{j \geq 1} \lambda_{j}(\Omega)^{2}=\sum_{j, k}\left|\left\langle T_{\Omega} s_{j}, s_{k}\right\rangle\right|^{2}=\sum_{j, k}\left|\int_{\Omega}\left\langle s_{j}, s_{k}\right\rangle\right|^{2}=\iint_{\Omega \times \Omega}|\Pi(x, y)|^{2} .
$$

Using (28) and (29) one may obtain some information on the distribution of the eigenvalues. This is done in the following lemma. 
Lemma 9. Let $0<\gamma<1$ and denote by $n(\Omega, \gamma)$ the number of eigenvalues $\lambda_{j}(\Omega)$ which are strictly greater than $\gamma$. Then we have the lower bound

$$
n(\Omega, \gamma) \geq \int_{\Omega}|\Pi(x, x)|-\frac{1}{1-\gamma} \iint_{\Omega \times \Omega^{c}}|\Pi(x, y)|^{2},
$$

and the upper bound

$$
n(\Omega, \gamma) \leq \int_{\Omega}|\Pi(x, x)|+\frac{1}{\gamma} \iint_{\Omega \times \Omega^{c}}|\Pi(x, y)|^{2}
$$

Proof. We have

$$
\mathbb{1}_{(\gamma, 1]}(x) \geq x-\frac{x(1-x)}{1-\gamma} \quad(0 \leq x \leq 1)
$$

hence

$$
n(\Omega, \gamma)=\sum_{j} \mathbb{1}_{(\gamma, 1]}\left(\lambda_{j}(\Omega)\right) \geq \sum_{j} \lambda_{j}(\Omega)-\frac{1}{1-\gamma} \sum_{j}\left(\lambda_{j}(\Omega)-\lambda_{j}(\Omega)^{2}\right) .
$$

Using (28), (29) and (7) now implies

$$
\begin{aligned}
n(\Omega, \gamma) & \geq \int_{\Omega}|\Pi(x, x)|-\frac{1}{1-\gamma}\left[\int_{\Omega}|\Pi(x, x)|-\iint_{\Omega \times \Omega}|\Pi(x, y)|^{2}\right] \\
& =\int_{\Omega}|\Pi(x, x)|-\frac{1}{1-\gamma}\left[\iint_{\Omega \times X}|\Pi(x, y)|^{2}-\iint_{\Omega \times \Omega}|\Pi(x, y)|^{2}\right],
\end{aligned}
$$

which proves (30). To prove (31) one may argue similarly using the inequality

$$
\mathbb{1}_{(\gamma, 1]}(x) \leq x+x(1-x) / \gamma \quad(0 \leq x \leq 1) .
$$

5.3. Now consider powers $L^{k}$ of the line bundle $L$. We obtain an operator $T_{\Omega}^{(k)}$ acting on $H^{0}\left(L^{k}\right)$ with eigenvalues

$$
\lambda_{1}^{(k)}(\Omega) \geq \lambda_{2}^{(k)}(\Omega) \geq \cdots \geq 0
$$

and we let $n_{k}(\Omega, \gamma)$ denote the number of eigenvalues strictly greater than $\gamma(0<\gamma<1)$.

Lemma 10. Let $\Lambda_{k}$ be a $\delta$-separated sampling set at level $k$ with sampling constants $A, B$. Then for any $z \in X$ and $r>0$,

$$
\#\left(\Lambda_{k} \cap B(z,(r+\delta) / \sqrt{k})\right) \geq n_{k}(B(z, r / \sqrt{k}), \gamma)
$$

where $\gamma$ is some constant lying between 0 and 1 such that $1 /(1-\gamma)$ is bounded by the sampling constant $B$ times a constant which may depend on $\delta$ but does not depend on $k, z, r$. 
Proof. Let $\left\{s_{j}\right\}$ be the orthonormal basis of $H^{0}\left(L^{k}\right)$ which is associated to the eigenvalues $\lambda_{j}^{(k)}(\Omega)$, where $\Omega=B(z, r / \sqrt{k})$. Let $N:=\#\left(\Lambda_{k} \cap B(z,(r+\delta / 2) / \sqrt{k})\right)$. We may restrict to the case when $N$ is strictly smaller than $\operatorname{dim} H^{0}\left(L^{k}\right)$, since otherwise the inequality holds trivially. In this case, we may choose a linear combination

$$
s=\sum_{j=1}^{N+1} c_{j} s_{j}
$$

of the first $N+1$ eigensections such that

$$
s(\lambda)=0, \quad \lambda \in \Lambda_{k} \cap B(x,(r+\delta / 2) / \sqrt{k}),
$$

and the $c_{j}$ are not all zero. Since $\Lambda_{k}$ is a sampling set, we have

$$
\|s\|^{2} \leq B k^{-n} \sum_{\lambda \in \Lambda_{k}}|s(\lambda)|^{2}=B k^{-n} \sum_{\lambda \in \Lambda_{k} \backslash B(x,(r+\delta / 2) / \sqrt{k})}|s(\lambda)|^{2} .
$$

Using the inequality (10) and the fact that $B(\lambda,(\delta / 2) / \sqrt{k})$ are disjoint balls, we get

$$
\|s\|^{2} \leq K B \sum_{\lambda} \int_{B(\lambda,(\delta / 2) / \sqrt{k})}|s|^{2} \leq K B \int_{X \backslash \Omega}|s|^{2},
$$

where the constant $K$ may depend on $\delta$ but does not depend on $k, z, r$. This implies

$$
\lambda_{N+1}(\Omega)\|s\|^{2}=\lambda_{N+1} \sum_{j=1}^{N+1}\left|c_{j}\right|^{2} \leq \sum_{j=1}^{N+1} \lambda_{j}\left|c_{j}\right|^{2}=\left\langle T_{\Omega}^{(k)} s, s\right\rangle=\int_{\Omega}|s|^{2} \leq \gamma\|s\|^{2},
$$

where $\gamma:=1-(K B)^{-1}$. This shows that $\lambda_{N+1}(\Omega) \leq \gamma$ and hence $n_{k}(\Omega, \gamma) \leq N$.

Lemma 11. Let $\Lambda_{k}$ be a $\delta$-separated interpolation set at level $k$ with interpolation constant $C$. Then for any $z \in X$ and $r>0$,

$$
\#\left(\Lambda_{k} \cap B(z,(r-\delta) / \sqrt{k})\right) \leq n_{k}(B(z, r / \sqrt{k}), \gamma)
$$

where $\gamma$ is some constant lying between 0 and 1 such that $1 / \gamma$ is bounded by the interpolation constant $C$ times a constant which may depend on $\delta$ but does not depend on $k, z, r$.

Proof. Let $W$ denote the orthogonal complement in $H^{0}\left(L^{k}\right)$ of the subspace of sections vanishing on $\Lambda_{k}$. Since $\Lambda_{k}$ is an interpolation set at level $k$, for any set $\left\{v_{\lambda}\right\}_{\lambda \in \Lambda_{k}}$, where each $v_{\lambda}$ is an element of the fiber of $\lambda$ in $L^{k}$, there is a section $s \in H^{0}\left(L^{k}\right)$ such that $s(\lambda)=v_{\lambda}\left(\lambda \in \Lambda_{k}\right)$ and

$$
\|s\|^{2} \leq C k^{-n} \sum_{\lambda \in \Lambda_{k}}|s(\lambda)|^{2} .
$$

By taking the orthogonal projection of $s$ onto $W$ we obtain another solution to the interpolation problem, which in addition belongs to $W$ (the projection neither changes the values of $s$ on $\Lambda_{k}$ nor increases its norm). 
On the other hand, a section in $W$ is uniquely determined by its values on $\Lambda_{k}$, as follows from the definition of $W$. Hence if $s$ is an arbitrary section in $W$, then it is the unique interpolant in $W$ to the values $\{s(\lambda)\}_{\lambda \in \Lambda_{k}}$. This implies that (32) holds for any $s \in W$.

Now let us denote by $x_{1}, \ldots, x_{N}$ the elements of $\Lambda_{k} \cap B(z,(r-\delta) / \sqrt{k})$. For each $1 \leq j \leq N$ we can find $s_{j} \in W$ such that $\left|s_{j}\left(x_{j}\right)\right|=1$ and $s_{j}$ vanishes on $\Lambda_{k} \backslash\left\{x_{j}\right\}$. Certainly, the $s_{j}$ form a linearly independent set of vectors. We denote by $F$ the $N$ dimensional linear subspace spanned by them.

Now take any $s \in F$. Then

$$
\|s\|^{2} \leq C k^{-n} \sum_{\lambda \in \Lambda_{k}}|s(\lambda)|^{2}=C k^{-n} \sum_{\lambda \in \Lambda_{k} \cap B(x,(r-\delta / 2) / \sqrt{k})}|s(\lambda)|^{2}<K C \int_{\Omega}|s|^{2},
$$

where $\Omega=B(z, r / \sqrt{k})$, and the constant $K$ may depend on $\delta$ but does not depend on $k, z, r$. The last inequality holds by (10) and the fact that $B(\lambda,(\delta / 2) / \sqrt{k})$ are disjoint balls. Hence

$$
\frac{\left\langle T_{\Omega}^{(k)} s, s\right\rangle}{\|s\|^{2}}=\frac{\int_{\Omega}|s|^{2}}{\|s\|^{2}}>\frac{1}{K C}=: \gamma,
$$

for any section $s$ in the $N$-dimensional linear subspace $F$. By the min-max theorem this implies that $\lambda_{N}(\Omega)>\gamma$ and hence $n_{k}(\Omega, \gamma) \geq N$.

\section{Curvature and density}

In the previous section we have used Landau's method to estimate the number of points of a sampling or interpolation set in a ball, where the estimate obtained was given in terms of the Bergman kernel $\Pi_{k}(x, y)$. In the present section we will prove Theorem 3 by relating the latter estimate to geometric properties of the positive line bundle $(L, \phi)$, namely, to the volume form associated with the curvature of the bundle.

6.1. Given a point $x \in X$, let $\xi_{1}, \ldots, \xi_{n}$ be a basis for the holomorphic cotangent space at $x$, orthonormal with respect to the hermitian metric $\omega$ on $X$. With respect to this basis, the form $\partial \bar{\partial} \phi$ is given at the point $x$ by

$$
\partial \bar{\partial} \phi=\sum_{j, k} \phi_{j, k} \xi_{j} \wedge \bar{\xi}_{k}
$$

where $\left(\phi_{j, k}\right)$ is a hermitian $n \times n$ matrix. The eigenvalues $\lambda_{1}(x), \ldots, \lambda_{n}(x)$ of this matrix are called the eigenvalues of the curvature form $\partial \bar{\partial} \phi$ with respect to the hermitian metric $\omega$.

Recall that the line bundle $L$ with metric $\phi$ is said to be positive if $i \partial \bar{\partial} \phi$ is a positive form. This is equivalent to all of the eigenvalues $\lambda_{1}(x), \ldots, \lambda_{n}(x)$ being strictly positive, for every $x \in X$. 
If the form $i \partial \bar{\partial} \phi$ is positive, then the $(n, n)$-form $(i \partial \bar{\partial} \phi)^{n}$ is a volume form on $X$. Our goal is to provide geometrical information on a sampling or interpolation array $\Lambda=\left\{\Lambda_{k}\right\}$, by relating the mass distribution of the measure

$$
k^{-n} \sum_{\lambda \in \Lambda_{k}} \delta_{\lambda}
$$

to the volume distribution of $(i \partial \bar{\partial} \phi)^{n}$ in a quantitative manner. We emphasize that the volume form $(i \partial \bar{\partial} \phi)^{n}$ is a characteristic of the hermitian metric $\phi$ on the line bundle only, and does not depend on the arbitrary hermitian metric $\omega$ that we have chosen on the manifold $X$. However, the curvature volume form $(i \partial \bar{\partial} \phi)^{n}$ is related to the volume form $V$ associated with $\omega$ through the eigenvalues, and we have

$$
(i \partial \bar{\partial} \phi)^{n}=n ! \lambda_{1}(x) \cdots \lambda_{n}(x) d V(x) .
$$

The eigenvalues of the curvature form are also related to the asymptotics of the Bergman function $\left|\Pi_{k}(x, x)\right|$. When the line bundle is positive, it was proven in [Tia90] (see [Ze198]) that

$$
\left|\Pi_{k}(x, x)\right|=\pi^{-n} \lambda_{1}(x) \cdots \lambda_{n}(x) k^{n}+O\left(k^{n-1}\right) .
$$

This a more precise result than (8). In fact, this is only the first term in a complete asymptotic expansion, obtained in [Ze198], into a power series in $k$ (see also [BBS08] for a different proof).

6.2. The main ingredient which we need for the proof of Theorem 3 is that the "error terms" in Landau's inequalities (26) and (27) are indeed small with respect to the main term. This is shown in the following lemma.

Lemma 12. Let the line bundle $(L, \phi)$ be positive. If $\Omega=B(z, r / \sqrt{k})$ with $z \in X$, then

$$
\iint_{\Omega \times \Omega^{c}}\left|\Pi_{k}(x, y)\right|^{2} \lesssim r^{2 n-1} .
$$

For the proof we will use the asymptotic off-diagonal estimate (9) for the Bergman kernel, which holds when the line bundle $(L, \phi)$ is positive. In fact, we do not need the precise exponential decay given by (9). It will be enough to use the fact that

$$
\left|\Pi_{k}(x, y)\right| \leq k^{n} \varphi(\sqrt{k} d(x, y)),
$$

where $\varphi$ is a smooth decreasing function on $[0, \infty)$ such that

$$
\varphi(u)=O\left(u^{-\alpha}\right) \quad \text { as } u \rightarrow \infty, \quad \text { for some } \alpha>n+1 / 2 .
$$

Proof of Lemma 12. We partition $\Omega$ into "dyadic shells" defined by

$$
\Omega_{j}:=\left\{x \in X:\left(1-2^{-j+1}\right) r / \sqrt{k} \leq d(x, z)<\left(1-2^{-j}\right) r / \sqrt{k}\right\} \quad(j \geq 1) .
$$


If $x \in \Omega_{j}$ and $y \in \Omega^{c}$ then $d(x, y)>2^{-j} r / \sqrt{k}$, and thus

$$
\iint_{\Omega \times \Omega^{c}}\left|\Pi_{k}(x, y)\right|^{2} \leq \sum_{j=1}^{\infty} \iint_{\Omega_{j} \times B\left(x, 2^{-j} r / \sqrt{k}\right)^{c}}\left|\Pi_{k}(x, y)\right|^{2} .
$$

To estimate the right hand side we use (35). For any $A>0$ we have

$$
\begin{array}{r}
\int_{B(x, A / \sqrt{k})^{c}}\left|\Pi_{k}(x, y)\right|^{2} d V(y)=\int_{0}^{\infty} V\left(\left\{y:\left|\Pi_{k}(x, y)\right|>\lambda\right\} \backslash B(x, A / \sqrt{k})\right) 2 \lambda d \lambda \\
\leq \int_{0}^{k^{n} \varphi(0)} V\left(\left\{y: \varphi(\sqrt{k} d(x, y)) \geq k^{-n} \lambda\right\} \backslash B(x, A / \sqrt{k})\right) 2 \lambda d \lambda .
\end{array}
$$

Since $\varphi$ is decreasing, the change of variable $\lambda=k^{n} \varphi(u)$ transforms the right hand side above to

$$
\begin{aligned}
& \int_{0}^{\infty} V(B(x, u / \sqrt{k}) \backslash B(x, A / \sqrt{k}))\left(2 k^{n} \varphi(u)\right)\left|k^{n} \varphi^{\prime}(u)\right| d u \\
& \lesssim \int_{A}^{\infty}(u / \sqrt{k})^{2 n}\left(2 k^{n} \varphi(u)\right)\left|k^{n} \varphi^{\prime}(u)\right| d u \lesssim k^{n} \int_{A}^{\infty} u^{2 n} \varphi(u)\left|\varphi^{\prime}(u)\right| d u .
\end{aligned}
$$

We also use an estimate for the volume of shells, namely

$$
V(B(x, \rho+\delta) \backslash B(x, \rho)) \lesssim \rho^{2 n-1} \delta \quad(0<\delta<\rho),
$$

which can be proved using the exponential map. In particular, this implies

$$
V\left(\Omega_{j}\right) \lesssim 2^{-j} r^{2 n} / k^{n}
$$

Combining all the estimates above yields

$$
\begin{aligned}
\iint_{\Omega \times \Omega^{c}}\left|\Pi_{k}(x, y)\right|^{2} & \lesssim \sum_{j=1}^{\infty}\left(2^{-j} r^{2 n} / k^{n}\right) k^{n} \int_{2^{-j} r}^{\infty} u^{2 n} \varphi(u)\left|\varphi^{\prime}(u)\right| d u \\
& =r^{2 n} \int_{0}^{\infty}\left[\sum_{j=1}^{\infty} 2^{-j} \mathbb{1}_{\left[2^{-j} r, \infty\right)}(u)\right] u^{2 n} \varphi(u)\left|\varphi^{\prime}(u)\right| d u \\
& \leq r^{2 n} \int_{0}^{\infty}(2 u / r) u^{2 n} \varphi(u)\left|\varphi^{\prime}(u)\right| d u \lesssim r^{2 n-1} \int_{0}^{\infty} u^{2 n} \varphi(u)^{2} d u
\end{aligned}
$$

where the integration by parts used is justified by (36). Since the last integral converges, again due to (36), this proves the lemma.

6.3. We can now finish the proof of Theorem 3 . It is an immediate consequence of the following result. 
Lemma 13. Let $(L, \phi)$ be positive. If $\Lambda_{k}$ be a $\delta$-separated sampling set at level $k$ with sampling constants $A, B$, then for any $z \in X$ and $r>0$,

$$
\frac{k^{-n} \#\left(\Lambda_{k} \cap \Omega\right)}{\int_{\Omega}(i \partial \bar{\partial} \phi)^{n}}>\frac{1}{\pi^{n} n !}-\frac{M}{r},
$$

where $\Omega=B(z, r / \sqrt{k})$, and the constant $M$ is bounded by the sampling constant $B$ times a constant which may depend on $\delta$ but does not depend on $k, z, r$.

Similarly, if $\Lambda_{k}$ is a $\delta$-separated interpolation set at level $k$ with interpolation constant $C$, then for any $z \in X$ and $r>0$,

$$
\frac{\left.k^{-n} \#\left(\Lambda_{k} \cap \Omega\right)\right)}{\int_{\Omega}(i \partial \bar{\partial} \phi)^{n}}<\frac{1}{\pi^{n} n !}+\frac{M}{r},
$$

where again $\Omega=B(z, r / \sqrt{k})$, and the constant $M$ is bounded by the interpolation constant $C$ times a constant which may depend on $\delta$ but does not depend on $k, z, r$.

Proof. Assume first that $\Lambda_{k}$ is a $\delta$-separated sampling set at level $k$. Let $\Omega=B(z, r / \sqrt{k})$. The separation condition together with (37) imply that the number of points of $\Lambda_{k}$ in the shell $B(z,(r+\delta) / \sqrt{k}) \backslash B(z, r / \sqrt{k})$ is less than $M_{1} r^{2 n-1}$. Hence by (26) and Lemma 12 we obtain

Using (33) and (34) now implies

$$
\#\left(\Lambda_{k} \cap \Omega\right) \geq \int_{\Omega}\left|\Pi_{k}(x, x)\right|-M_{2} r^{2 n-1} .
$$

$$
\#\left(\Lambda_{k} \cap \Omega\right) \geq \frac{k^{n}}{\pi^{n} n !} \int_{\Omega}(i \partial \bar{\partial} \phi)^{n}-M_{2} r^{2 n-1}-M_{3} k^{n-1} V(\Omega) .
$$

Since $V(\Omega) \lesssim r^{2 n} / k^{n}$ and $r / \sqrt{k} \leq \operatorname{diam}(X)$ it follows that

$$
\#\left(\Lambda_{k} \cap \Omega\right) \geq \frac{k^{n}}{\pi^{n} n !} \int_{\Omega}(i \partial \bar{\partial} \phi)^{n}-M_{4} r^{2 n-1},
$$

and since $k^{n} \int_{\Omega}(i \partial \bar{\partial} \phi)^{n}$ is of order $r^{2 n}$, this proves the claimed inequality. In the second case, when $\Lambda_{k}$ is a $\delta$-separated interpolation set at level $k$, the result is proved in a similar way using (27) instead of (26).

This concludes the proof of Theorem 3.

Remark 2. One may also define sampling and interpolation arrays with respect to the $L^{p}$ norm on the line bundle $(1 \leq p \leq \infty)$. The necessary density conditions given in Corollary 1 could be extended to this setting as well. This is rather standard and we do not discuss the details (see e.g. [Mar07]).

\section{Equidistribution of Fekete points}

In this section we estimate from above and below the number of Fekete points that lie in a ball. Our proof is inspired by the work of Nitzan and Olevskii [NO12] who provide a new proof of Landau's necessary density condition for sampling and interpolation in the Paley-Wiener space. Their main idea, which we adapt to the study of Fekete points, 
is to find a discrete representation of the Bergman kernel on the diagonal as a linear combination of reproducing kernels on the Fekete points. This produces a "tessellation" by functions concentrated around the Fekete points. The same technique can be used to provide an upper bound for the Kantorovich-Wasserstein distance between the Fekete measure (1) and its limiting measure. We also use the Fekete points to construct a sampling or interpolation array with density arbitrarily close to the critical one, showing that the necessary density conditions in Corollary 1 are sharp.

7.1. To prove Theorems 1 and 2 we will need two lemmas. The first one is an $L^{1}$-variant of the off-diagonal decay estimate of the Bergman kernel.

Lemma 14. Let the line bundle $(L, \phi)$ be positive. Then

(i) $\sup _{x \in X} \int_{X}\left|\Pi_{k}(x, y)\right| d V(y) \lesssim 1$;

(ii) if $\Omega=B(z, R / \sqrt{k})$ then

$$
k^{n} \iint_{\Omega \times \Omega^{c}}\left|\Pi_{k}(x, y)\right| \lesssim R^{2 n-1}
$$

uniformly in $z \in X$;

(iii) $\sup _{x \in X} \int_{X} d(x, y)\left|\Pi_{k}(x, y)\right| d V(y) \lesssim 1 / \sqrt{k}$.

This can be proved with an argument completely similar to the one used in the proof of Lemma 12, so we omit the details.

Lemma 15. Let $\left\{\ell_{\lambda}\right\}$ be the Lagrange sections associated to the Fekete points $\mathcal{F}_{k}$. Then there exist sections $\Phi_{\lambda} \in H^{0}\left(L^{k}\right), \lambda \in \mathcal{F}_{k}$, such that:

(i) $\int_{X}\left\langle\ell_{\lambda}(x), \Phi_{\lambda}(x)\right\rangle d V(x)=1$ for all $\lambda \in \mathcal{F}_{k}$.

(ii) $\sum_{\lambda \in \mathcal{F}_{k}}\left\langle\ell_{\lambda}(x), \Phi_{\lambda}(x)\right\rangle=\left|\Pi_{k}(x, x)\right|$ for all $x \in X$.

(iii) $\left|\Phi_{\lambda}(x)\right|=\left|\Pi_{k}(x, \lambda)\right|$ for all $x \in X$ and $\lambda \in \mathcal{F}_{k}$.

Proof. Let $s_{1}, \ldots, s_{N}$ be an orthonormal basis for $H^{0}\left(L^{k}\right)$. Let $e_{\lambda}(x)$ be a holomorphic frame in a neighborhood $U_{\lambda}$ of $\lambda\left(\lambda \in \mathcal{F}_{k}\right)$. Then

$$
s_{j}(x)=f_{j, \lambda}(x) e_{\lambda}(x), \quad x \in U_{\lambda} .
$$

By Lemma 1, if we define

$$
\Phi_{\lambda}(x):=\left|e_{\lambda}(\lambda)\right| \sum_{j=1}^{N} \overline{f_{j, \lambda}(\lambda)} s_{j}(x)
$$

then (iii) is satisfied.

We now choose $e_{\lambda}(x):=\ell_{\lambda}(x)$, the Lagrange section. Then, since $\left|\ell_{\lambda}(\lambda)\right|=1$, we have

$$
\Phi_{\lambda}(x)=\sum_{j=1}^{N} \overline{f_{j, \lambda}(\lambda)} s_{j}(x) .
$$

Since $\left\{s_{j}(x)\right\}$ is an orthonormal basis,

$$
\ell_{\lambda}=\sum_{j=1}^{N} s_{j} \int_{X}\left\langle\ell_{\lambda}(x), s_{j}(x)\right\rangle d V(x) .
$$


Therefore

$$
\ell_{\lambda}(\lambda)=\sum_{j=1}^{N} s_{j}(\lambda) \int_{X}\left\langle\ell_{\lambda}(x), s_{j}(x)\right\rangle d V(x)=\sum_{j=1}^{N} f_{j, \lambda} \ell_{\lambda}(\lambda) \int_{X}\left\langle\ell_{\lambda}(x), s_{j}(x)\right\rangle d V(x) .
$$

Thus,

$$
1=\int_{X}\left\langle\ell_{\lambda}(x), \sum_{j=1}^{N} \overline{f_{j, \lambda(\lambda)}} s_{j}(x)\right\rangle d V(x)=\int_{X}\left\langle\ell_{\lambda}(x), \Phi_{\lambda}(x)\right\rangle d V(x),
$$

which gives (i).

Since $\left\{\ell_{\lambda}\right\}$ is a "Lagrange basis" for $H^{0}\left(L^{k}\right)$,

$$
s_{j}=\sum_{\lambda \in \mathcal{F}_{k}}\left\langle s_{j}(\lambda), \ell_{\lambda}(\lambda)\right\rangle \ell_{\lambda}=\sum_{\lambda \in \mathcal{F}_{k}}\left\langle f_{j, \lambda}(\lambda) \ell_{\lambda}(\lambda), \ell_{\lambda}(\lambda)\right\rangle \ell_{\lambda}=\sum_{\lambda \in \mathcal{F}_{k}} f_{j, \lambda}(\lambda) \ell_{\lambda} .
$$

Therefore

$$
\begin{aligned}
\left|\Pi_{k}(x, x)\right| & =\sum_{j=1}^{N}\left|s_{j}(x)\right|^{2}=\sum_{j=1}^{N}\left\langle s_{j}(x), s_{j}(x)\right\rangle=\sum_{j=1}^{N}\left\langle\sum_{\lambda \in \mathcal{F}_{k}} f_{j, \lambda}(\lambda) \ell_{\lambda}(x), s_{j}(x)\right\rangle \\
& =\sum_{\lambda \in \mathcal{F}_{k}}\left\langle\ell_{\lambda}(x), \sum_{j=1}^{N} \overline{f_{j, \lambda}(\lambda)} s_{j}(x)\right\rangle=\sum_{\lambda \in \mathcal{F}_{k}}\left\langle\ell_{\lambda}(x), \Phi_{\lambda}(x)\right\rangle,
\end{aligned}
$$

which gives (ii).

Proof of Theorem 1. Denote $\Omega:=B(z, R / \sqrt{k})$. By Lemma 15 we have

$$
\begin{aligned}
\#\left(\mathcal{F}_{k} \cap \Omega\right)- & \int_{\Omega}\left|\Pi_{k}(x, x)\right|=\sum_{\lambda \in \mathcal{F}_{k} \cap \Omega} \int_{X}\left\langle\ell_{\lambda}(x), \Phi_{\lambda}(x)\right\rangle-\int_{\Omega} \sum_{\lambda \in \mathcal{F}_{k}}\left\langle\ell_{\lambda}(x), \Phi_{\lambda}(x)\right\rangle \\
& =\int_{X \backslash \Omega} \sum_{\lambda \in \mathcal{F}_{k} \cap \Omega}\left\langle\ell_{\lambda}(x), \Phi_{\lambda}(x)\right\rangle-\int_{\Omega} \sum_{\lambda \in F_{k} \cap(X \backslash \Omega)}\left\langle\ell_{\lambda}(x), \Phi_{\lambda}(x)\right\rangle=A_{1}-A_{2} .
\end{aligned}
$$

We first estimate $A_{1}$. We have

$$
\left|A_{1}\right| \leq \int_{\Omega^{c}} \sum_{\lambda \in \mathcal{F}_{k} \cap \Omega}\left|\Pi_{k}(x, \lambda)\right| .
$$

By the sub-mean value property (10),

$$
\left|\Pi_{k}(x, \lambda)\right| \lesssim(\delta / \sqrt{k})^{-2 n} \int_{B(\lambda, \delta / \sqrt{k})}\left|\Pi_{k}(x, y)\right| d V(y) .
$$

If we take $\delta$ to be the separation constant of $\mathcal{F}_{k}$ (Lemma 3), then

$$
\sum_{\lambda \in \mathcal{F}_{k} \cap B(z,(R-\delta) / \sqrt{k})}\left|\Pi_{k}(x, \lambda)\right| \lesssim k^{n} \int_{\Omega}\left|\Pi_{k}(x, y)\right| d V(y) .
$$


Hence by part (ii) of Lemma 14,

$$
\int_{\Omega^{c}} \sum_{\lambda \in \mathcal{F}_{k} \cap B(z,(R-\delta) / \sqrt{k})}\left|\Pi_{k}(x, \lambda)\right| \lesssim k^{n} \iint_{\Omega^{c} \times \Omega}\left|\Pi_{k}(x, y)\right| \lesssim R^{2 n-1} .
$$

On the other hand, the separation condition together with (37) imply

$$
\#\left(\mathcal{F}_{k} \cap(B(z, R / \sqrt{k}) \backslash B(z, R-\delta / \sqrt{k}))\right) \lesssim R^{2 n-1},
$$

and hence

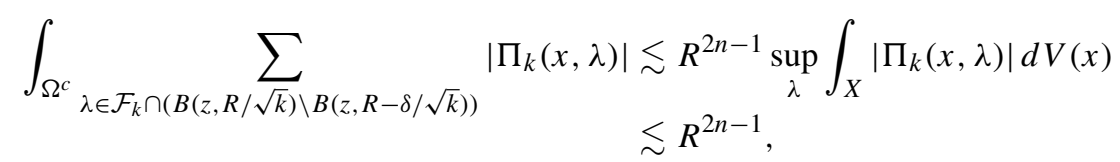

by Lemma 14(i). Combining the two estimates yields $\left|A_{1}\right| \lesssim R^{2 n-1}$. In the same way, we can also get the estimate $\left|A_{2}\right| \lesssim R^{2 n-1}$. Hence from (33) and (34),

$$
\#\left(\mathcal{F}_{k} \cap \Omega\right)=\int_{\Omega}\left|\Pi_{k}(x, x)\right|+O\left(R^{2 n-1}\right)=\left(1+O\left(R^{-1}\right)\right) \frac{k^{n}}{\pi^{n} n !} \int_{\Omega}(i \partial \bar{\partial} \phi)^{n} .
$$

We also infer from (6) that

$$
\# \mathcal{F}_{k}=\operatorname{dim} H^{0}\left(L^{k}\right)=\int_{X}\left|\Pi_{k}(x, x)\right|=\left(1+O\left(k^{-1}\right)\right) \frac{k^{n}}{\pi^{n} n !} \int_{X}(i \partial \bar{\partial} \phi)^{n} .
$$

Since we may assume $R / \sqrt{k} \leq \operatorname{diam}(X)$, combining (40) with (41) proves the theorem.

7.2. The estimate (40) for the number of Fekete points in a ball shows, in particular, that a Fekete array $\left\{\mathcal{F}_{k}\right\}$ for the positive line bundle has the critical density,

$$
D^{-}\left(\left\{\mathcal{F}_{k}\right\}\right)=D^{+}\left(\left\{\mathcal{F}_{k}\right\}\right)=\frac{1}{\pi^{n} n !} .
$$

It is easy to check that the density of the perturbed array $\left\{\mathcal{F}_{(1 \pm \varepsilon) k}\right\}$ will be the critical value multiplied by $(1 \pm \varepsilon)^{n}$. Combining this with Corollary 2 shows that the density threshold in Corollary 1 is sharp.

Corollary 3. Let $(L, \phi)$ be positive. Then:

(i) For any $\varepsilon>0$ there is a sampling array $\Lambda$ with $D^{+}(\Lambda)<1 /\left(\pi^{n} n !\right)+\varepsilon$.

(ii) For any $\varepsilon>0$ there is an interpolation array $\Lambda$ with $D^{-}(\Lambda)>1 /\left(\pi^{n} n !\right)-\varepsilon$. 
7.3. Given two probability measures $\mu$ and $v$ on a metric space $X$, one defines their Kantorovich-Wasserstein distance as

$$
W(\mu, v)=\inf \left\{\iint_{X \times X} \operatorname{dist}(x, y) d \rho(x, y)\right\}
$$

where the infimum is taken over all Borel probability measures $\rho$ on $X \times X$ with marginals $\rho(\cdot, X)=\mu$ and $\rho(X, \cdot)=v$. This metric plays a key role in transportation problems (see for instance [Vil09]).

In our setting we have two probability measures: the first one is the Fekete measure $\mu_{k}$ defined in (1), and the second is the measure $(i \partial \bar{\partial} \phi)^{n}$ normalized to have total mass 1 , which we denote by $v$. It is known (see [Blü90] for instance) that on a Riemannian manifold, if $\mu_{k}(B(x, r)) \rightarrow v(B(x, r))$ for all balls, as guaranteed by Theorem 1, then $\mu_{k}$ converges weakly to $v$ as $k \rightarrow \infty$, where the latter means that $\int f d \mu_{k} \rightarrow \int f d \nu$ for any continuous function $f$ on $X$.

The Kantorovich-Wasserstein distance metrizes the weak convergence of measures. Here we prove Theorem 2 which describes the rate of convergence in the KantorovichWasserstein distance. For the proof it will be convenient to recall the dual formulation (see [Vil09, formula (6.3)])

$$
W(\mu, v)=\sup \left\{\left|\int_{X} f d(\mu-v)\right|: f \in \operatorname{Lip}_{1,1}(X)\right\},
$$

where $\operatorname{Lip}_{1,1}(X)$ is the collection of all functions $f$ on $X$ satisfying $|f(x)-f(y)| \leq$ $d(x, y)$.

Proof of Theorem 2. To prove the lower bound for the Kantorovich-Wasserstein distance we consider the function $f_{k}(x)=\operatorname{dist}\left(x, \mathcal{F}_{k}\right)$. Then clearly $f_{k} \in \operatorname{Lip}_{1,1}(X)$, and moreover $f_{k}$ vanishes on $\mathcal{F}_{k}$. Hence by (42),

$$
W\left(\mu_{k}, v\right) \geq\left|\int_{X} f_{k}\left(d \mu_{k}-d v\right)\right|=\int_{X} f_{k} d v .
$$

The function $f_{k}$ is bounded below by $\delta>0$ outside the balls $B(\lambda, \delta), \lambda \in \mathcal{F}_{k}$, and so

$$
\int_{X} f_{k} d v \geq \delta \cdot v\left(X \backslash \bigcup_{x \in \mathcal{F}_{k}} B(x, \delta)\right) \geq \delta\left(1-C \delta^{2 n} \# \mathcal{F}_{k}\right) .
$$

We choose $\delta=\delta(k)$ such that $C \delta^{2 n} \# \mathcal{F}_{k}=1 / 2$. Since $\# \mathcal{F}_{k} \simeq k^{n}$ by (3), this implies

$$
W\left(\mu_{k}, v\right) \gtrsim k^{-1 / 2} \text {. }
$$

For the upper estimate we will use the following alternative definition of the Kantorovich-Wasserstein distance which is equivalent to the original one:

$$
W(\mu, v)=\inf _{\rho \in S} \iint_{X \times X} \operatorname{dist}(x, y)|d \rho(x, y)|
$$


where the infimum is now taken over the set $S$ of all complex measures $\rho$ on $X \times X$ with marginals $\rho(\cdot, X)=\mu$ and $\rho(X, \cdot)=v$. In order to prove (43) recall the dual formulation (42). Now, for any complex measure $\rho$ with marginals $\mu$ and $v$ we have

$$
\left|\int_{X} f d(\mu-v)\right|=\left|\iint_{X \times X}(f(x)-f(y)) d \rho(x, y)\right| \leq \iint_{X \times X} \operatorname{dist}(x, y)|d \rho(x, y)| .
$$

Therefore

$$
W(\mu, \nu) \leq \inf _{\rho \in S} \iint_{X \times X} \operatorname{dist}(x, y)|d \rho(x, y)|,
$$

the other inequality being trivial.

We will first prove that $W\left(\mu_{k}, v_{k}\right) \lesssim 1 / \sqrt{k}$ where $\mu_{k}$ is the Fekete measure defined in (1) and $v_{k}$ is the probability measure defined as

$$
d \nu_{k}(y):=\frac{1}{N_{k}}\left|\Pi_{k}(y, y)\right| d V(y),
$$

where $N_{k}=\# \mathcal{F}_{k}$. This is a probability measure because of (6). To get an upper bound for $W\left(\mu_{k}, v_{k}\right)$ we choose a complex measure $\rho$ as

$$
d \rho(x, y):=\frac{1}{N_{k}} \sum_{\lambda \in \mathcal{F}_{k}} \delta_{\lambda}(x) \times\left\langle\ell_{\lambda}(y), \Phi_{\lambda}(y)\right\rangle d V(y)
$$

where $\Phi_{\lambda}$ are the sections defined in Lemma 15 and $\ell_{\lambda}$ are the Lagrange sections. Observe that Lemma 15(i) implies that $\rho(\cdot, X)=\mu_{k}$, and Lemma 15(ii) yields $\rho(X, \cdot)=v_{k}$. Thus

$$
\begin{aligned}
W\left(\mu_{k}, v_{k}\right) & \leq \iint_{X \times X} \operatorname{dist}(x, y)|d \rho(x, y)| \\
& =\frac{1}{N_{k}} \sum_{\lambda \in \mathcal{F}_{k}} \int_{X} \operatorname{dist}(\lambda, y)\left|\left\langle\ell_{\lambda}(y), \Phi_{\lambda}(y)\right\rangle\right| d V(y) .
\end{aligned}
$$

We know that by the definition of Fekete points, the Lagrange sections are bounded and $\left|\ell_{\lambda}(y)\right| \leq 1$ (see (13)), and moreover $\left|\Phi_{\lambda}(y)\right|=\left|\Pi_{k}(y, \lambda)\right|$ (Lemma 15(iii)). Therefore,

$$
W\left(\mu_{k}, v_{k}\right) \leq \frac{1}{N_{k}} \sum_{\lambda \in \mathcal{F}_{k}} \int_{X} \operatorname{dist}(\lambda, y)\left|\Pi_{k}(y, \lambda)\right| d V(y) \lesssim 1 / \sqrt{k},
$$

where we have used the estimates of Lemma 14(iii). Finally, if we denote by $v$ the measure $(i \partial \bar{\partial} \phi)^{n}$ divided by its total mass, we observe that $W\left(v_{k}, v\right) \lesssim 1 / k$ since the total variation $\left\|v_{k}-v\right\|$ is $\lesssim 1 / k$, by (33) and (34), and since the total variation controls the Kantorovich-Wasserstein distance (see [Vil09, Theorem 6.15]). We have thus proved that $W\left(\mu_{k}, v\right) \lesssim 1 / \sqrt{k}$ as desired. 


\section{Simultaneously sampling and interpolation arrays}

8.1. In this section we assume that $X$ is a projective manifold, but we work with a metric $\phi$ on the line bundle $L$ which is only semipositive. We will show that if there is a point in $X$ where $\phi$ has a strictly positive curvature, then sections of high powers of the line bundle closely resemble functions in the Bargmann-Fock space. This observation will allow us to establish Theorem 5, showing that in this case there are no arrays which are simultaneously sampling and interpolation for $(L, \phi)$. The non-existence of simultaneously sampling and interpolation sequences is a recent result in the classical Bargmann-Fock space [AFK14, GM13].

Actually we could have replaced the assumption that $X$ is projective by the apparently weaker condition that $X$ is a Kähler manifold. However, the solution of Siu [Siu84] to the Grauert-Riemenschneider conjecture shows that, under the hypothesis that $L$ is semipositive with a point where it has a strictly positive curvature, the base manifold $X$ is Moishezon, and being also Kähler it is automatically projective [Mő̌66].

The proof of Siu also shows that under the hypothesis of the theorem, $L$ is big and thus there is a strictly positive singular metric $\phi_{s}$ on $L$ that is in $L_{\text {loc }}^{1}$ and smooth at all points of $X$ outside a proper analytic set $E$ (see [MM07, Theorem 2.3.30]).

8.2. We fix a point $x_{0} \in X \backslash E$ where the original metric on $L$ has positive curvature.

Definition 3. We say that we have normalized coordinates in a neighborhood of $x_{0} \in$ $X \backslash E$ if we have a coordinate chart that is mapped to a neighborhood of 0 in $\mathbb{C}^{n}$ and a local holomorphic frame $e_{L}(z)$ such that the following conditions hold:

- the curvature form of the line bundle at $x_{0}$ is given by $\Theta(0)=\sum_{j=1}^{n} d z_{j} \wedge d \bar{z}_{j}$;

- $h(0)=1$ and $\frac{\partial h}{\partial z_{j}}(0)=\frac{\partial^{2} h}{\partial z_{j} \partial z_{k}}(0)=0$;

where $h(z)=\left|e_{L}(z)\right|^{2}$, and $\Theta(z)=-\partial \bar{\partial} \log h(z)$ is the curvature form.

This can always be arranged if the curvature of $h$ is smooth and positive at the point $x_{0}$, by choosing appropriate coordinates and a convenient local frame. Observe that in normalized coordinates,

$$
h(z)=e^{-|z|^{2}+o\left(|z|^{2}\right)} .
$$

We now fix a neighborhood $B(0, \delta)$ of the origin in $\mathbb{C}^{n}$ that is mapped by normal coordinates to a neighborhood $U$ of $x_{0}$ in $X$.

Definition 4. We define the sets $\Sigma_{k} \subset \mathbb{C}^{n}$ as follows: $\sigma \in \Sigma_{k}$ if and only if $\sigma / \sqrt{k}$ is mapped by the normal coordinates to a point in $\Lambda_{K} \cap U$. By definition $\Sigma_{k} \subset B(0, \delta \sqrt{k})$.

If $\Lambda_{k}$ is both an interpolation and sampling array, we will construct a sequence $\Sigma \subset \mathbb{C}^{n}$ such that it is both interpolation and sampling for the Bargmann-Fock space.

Definition 5. Given $p \in[1, \infty)$, the Bargmann-Fock space $\mathcal{B F}^{p}$ consists of all entire functions such that

$$
\|f\|_{p}^{p}:=\int_{\mathbb{C}^{n}}|f(z)|^{p} e^{-p|z|^{2} / 2} d m(z)<\infty .
$$


When $p=\infty$ the natural norm is

$$
\|f\|_{\infty}:=\sup _{\mathbb{C}^{n}}|f(z)| e^{-|z|^{2} / 2}
$$

A sequence $\Sigma$ is sampling for the Bargmann-Fock space $\mathcal{B} \mathcal{F}^{2}$ if and only if

$$
\|f\|_{2}^{2} \lesssim \sum_{\sigma}|f(\sigma)|^{2} e^{-|\sigma|^{2}} \lesssim\|f\|_{2}^{2}
$$

and it is interpolation for $\mathcal{B F}^{2}$ if given any values $\left\{v_{\sigma}\right\}$ there is a function $f \in \mathcal{B F}^{2}$ such that $f(\sigma)=v_{\sigma}$ and

$$
\|f\|_{2}^{2} \lesssim \sum_{\sigma}\left|v_{\sigma}\right|^{2} e^{-|\sigma|^{2}}
$$

provided that the right hand side is finite.

It is known (see [AFK14] and [GM13]), that there do not exist sequences that are simultaneously sampling and interpolation in $\mathcal{B F}^{2}\left(\mathbb{C}^{n}\right)$.

The key ingredient in the construction of $\Sigma$ is that sections of high powers of the (locally positive) line bundle behave as functions in the Bargmann-Fock space when properly rescaled. This is a well known phenomenon that can be illustrated by the fact that the Bergman kernel universally converges to the Bergman kernel of the Bargmann-Fock space in normal coordinates if rescaled properly (see [BSZ00]). The next theorem is another illustration of the same fact. In order to state it we need the notion of weak limits of sequences. If we have a collection of separated sequences $\Sigma_{k} \subset \mathbb{C}^{n}$ with a uniform separation constant for all $k$ and another separated sequence $\Sigma \subset \mathbb{C}^{n}$, we say that $\Sigma_{k}$ converges weakly to $\Sigma$ if the corresponding measures $\mu_{k}=\sum_{\sigma \in \Sigma_{k}} \delta_{\sigma_{k}}$ converge weakly to $\sum_{\sigma \in \Sigma} \delta_{\sigma}$. This notion was used extensively by Beurling in his study of sampling sequences in the Paley-Wiener space and it will also be useful in our context.

Theorem 6. Let $\Lambda_{k}$ be a separated sampling array for $L^{k}$ and let $\Sigma$ be any weak limit of a subsequence of $\Sigma_{k}$. Then $\Sigma$ is a sampling sequence for $\mathcal{B F}^{2}\left(\mathbb{C}^{n}\right)$.

Let $\Lambda_{k}$ be an interpolation array for $L^{k}$ and let $\Sigma$ be any weak limit of a subsequence of $\Sigma_{k}$. Then $\Sigma$ is an interpolation sequence for $\mathcal{B F}^{2}\left(\mathbb{C}^{n}\right)$.

Proof. Let us start by the interpolation part. Assume that $\Sigma$ is the weak limit of a subsequence of $\Sigma_{k}$ that, with an abuse of notation, will be still denoted by $\Sigma_{k}$. Let us take a sequence $\left\{v_{\sigma}\right\}_{\sigma \in \Sigma}, v_{\sigma} \in \mathbb{C}$, with $\sum_{\sigma \in \Sigma}\left|v_{\sigma}\right|^{2} e^{-|\sigma|^{2}}<\infty$. We are going to construct a sequence of functions $f_{k} \in \mathcal{H}\left(B\left(0, M_{k}\right)\right)$ with $M_{k} \rightarrow \infty$ such that

$$
\sup _{k} \int_{|z|<M_{k}}\left|f_{k}(z)\right|^{2} e^{-|z|^{2}} d m(z)<\infty,
$$

and $\lim _{k} f_{k}(\sigma)=v_{\sigma}$ for all $\sigma \in \Sigma$. Then by a normal family argument we conclude that there is an interpolating function $f \in \mathcal{B F}^{2}$ with $f(\sigma)=v_{\sigma}$. Actually we may assume 
without loss of generality that, except for a finite number of points, $v_{\sigma}=0$. This is harmless if

$$
\limsup _{k \rightarrow \infty} \int_{|z|<M_{k}}\left|f_{k}(z)\right|^{2} e^{-|z|^{2}} d m(z) \leq C \sum_{\sigma}\left|v_{\sigma}\right|^{2} e^{-|\sigma|^{2}}
$$

with $C$ a constant independent of the number of non-zero terms.

Since we are assuming that the metric is smooth, and we are using normalized coordinates, we can use Definition 3 and find an increasing sequence $M_{k}$ with $\lim M_{k} \rightarrow \infty$ (but with $M_{k} / \sqrt{k} \rightarrow 0$ ) such that around $x_{0}, h(z)^{k} \simeq e^{-k|z|^{2}}$ for all $|z|<M_{k} / \sqrt{k}$.

Take some given values $v_{\sigma}$. We denote by $\Sigma^{\prime} \subset \Sigma$ the finite set of points $\sigma \in \Sigma$ such that $v_{\sigma} \neq 0$. For $k$ large enough, $|\sigma / \sqrt{k}|<M_{k}$ for all $\sigma \in \Sigma^{\prime}$. For $\sigma \in \Sigma^{\prime}$ there is an associated $\lambda_{\sigma}^{k} \in \Lambda_{k}$ such that $\sqrt{k} \lambda_{\sigma}^{k} \rightarrow \sigma$ because $\Sigma_{k} \rightarrow \Sigma$ weakly (here we are identifying the points in $\mathbb{C}^{n}$ and in $X$ by its coordinate chart). Consider the interpolation problem with data $v_{\sigma} e_{L}^{k}$ at the points $\lambda_{\sigma}^{k}, \sigma \in \Sigma^{\prime}$. By hypothesis there is a section $s \in$ $H^{0}\left(L^{k}\right)$ such that $s_{k}\left(\lambda_{\sigma}^{k}\right)=v_{\sigma} e_{L}^{k}\left(\lambda_{\sigma}^{k}\right)$ and

$$
\left\|s_{k}\right\|^{2} \leq \frac{C}{k^{n}} \sum_{\sigma \in \Sigma^{\prime}}\left|v_{\sigma}\right|^{2} h\left(\lambda_{\sigma}^{k}\right)^{k} .
$$

Near $x_{0}$ we may write $s_{k}(z)=g_{k}(z) e_{L}^{k}(z)$ and thus

$$
\begin{aligned}
\int_{|z| \leq M_{k} / \sqrt{k}}\left|g_{k}(z)\right|^{2} e^{-k|z|^{2}} d m(z) & \lesssim\left\|s_{k}\right\|^{2} \leq \frac{C}{k^{n}} \sum_{\sigma \in \Sigma^{\prime}}\left|v_{\sigma}\right|^{2} h\left(\lambda_{\sigma}^{k}\right)^{k} \\
& \leq \frac{C}{k^{n}} \sum_{\sigma \in \Sigma^{\prime}}\left|v_{\sigma}\right|^{2} e^{-k\left|\lambda_{\sigma}^{k}\right|^{2}} .
\end{aligned}
$$

The functions $f_{k}(z)=g_{k}(\sqrt{k} z)$ are holomorphic in $|z|<M_{k}$ and they satisfy

$$
\int_{|z|<M_{k}}\left|f_{k}(z)\right|^{2} e^{-|z|^{2}} \leq C \sum_{\sigma \in \Sigma^{\prime}}\left|v_{\sigma}\right|^{2} e^{-\left|\sqrt{k} \lambda_{\sigma}^{k}\right|^{2}} .
$$

If we let $k \rightarrow \infty$ on the right hand side of the inequality, we obtain

$$
\limsup _{k \rightarrow \infty} \int_{|z|<M_{k}}\left|f_{k}(z)\right|^{2} e^{-|z|^{2}} \lesssim \sum_{\sigma \in \Sigma^{\prime}}\left|v_{\sigma}\right|^{2} e^{-|\sigma|^{2}}
$$

8.3. The sampling part of Theorem 6 is slightly more involved. We need an approximation lemma that in an informal way shows that one can locally approximate functions in the Bargmann-Fock space by sections of $L^{k}$. More precisely, we will work with semipositive holomorphic line bundles $L$ over a projective manifold $X$ that have some point where the metric on $L$ has strictly positive curvature. As mentioned before, such bundles are big line bundles and therefore they admit a strictly positive singular metric $\phi_{S}$ that is in $L_{\text {loc }}^{1}$ and is smooth away from an analytic exceptional set $E \subset X$ (see [MM07, Theorem 2.3.30]). 
Lemma 16. Let $L$ be a semipositive holomorphic line bundle over a projective manifold $X$ with some point where the metric on $L$ has positive curvature. Fix a point $x_{0} \in X$ where the metric has strictly positive curvature and such that $x_{0}$ is not contained in the exceptional analytic set $E$, and consider normal coordinates around $x_{0}$ and the corresponding frame e $(z)$. Given any function $f$ in the Bargmann-Fock space, and any large $M>0$, there is a $k_{0} \in \mathbb{N}$ such that for all $k \geq k_{0}$ there are global holomorphic sections $s_{k}(z)=f_{k}(z) e_{k}(z)$ of $L^{k}$ such that in the normalized coordinates around $x_{0}$,

$$
\int_{|z|<M / \sqrt{k}}\left|f(\sqrt{k} z)-f_{k}(z)\right|^{2} e^{-k|z|^{2}} d z \lesssim \frac{1}{M^{2}}\|f\|^{2} / k^{n}
$$

and

$$
\int_{|z|>M / \sqrt{k}}\left|s_{k}\right|_{\phi}^{2} \lesssim \frac{1}{M^{2}}\|f\|^{2} / k^{n}
$$

In particular $\left\|s_{k}\right\|^{2} \simeq\|f\|^{2} / k^{n}$ for all $k \geq k_{0}$.

Thus, in a sense, $s_{k}$ are global sections that approximate $f$ around $x$.

This lemma follows from the $L^{2} \bar{\partial}$-estimates on line bundles for singular metrics. This is a refinement of Hörmander's theorem that is due to Demailly-Nadel (see [Ber10] for a nice exposition). We will use the following theorem.

Theorem 7 (Demailly-Nadel). Let $X$ be a projective manifold. Let $L$ be a holomorphic line bundle over $X$ which has a possibly singular metric $\phi_{s}$ whose curvature satisfies

$$
i \partial \bar{\partial} \phi_{s} \geq \varepsilon \omega,
$$

where $\omega$ is a Kähler form. Let $f$ be an $L$-valued $\bar{\partial}$-closed form of bidegree $(n, 1)$. Then there is a solution $u$ to the equation $\bar{\partial} u=f$ satisfying

$$
\|u\|_{\omega, \phi_{s}}^{2} \lesssim \int_{X}|f|_{\partial \bar{\partial} \phi_{s}}^{2} e^{-\phi_{s}} .
$$

In this statement $|f(x)|_{\partial \bar{\partial} \phi_{s}}$ is the pointwise norm on $(n, 1)$-forms induced by the singular Hermitian metric in $X$. In particular if $i \partial \bar{\partial} \phi_{s} \geq M \omega$ on the support of $f$, then

$$
\|u\|_{\omega, \phi_{s}}^{2} \lesssim \frac{1}{M}\|f\|_{\omega, \phi_{s}}^{2} .
$$

We now prove the approximation lemma.

Proof of Lemma 16. Let $\chi$ be a cutoff function supported in a ball of radius $M$ centered at the origin and equal to 1 in $B(0, M / 2)$. We take $M$ so large that $|\nabla \chi| \leq 4 / M$. We set $\chi_{k}(z)=\chi(z \sqrt{k})$. We define, in normal coordinates, $g_{k}(z)=f(\sqrt{k} z) \chi_{k}(z) e_{k}(z)$. The section $g_{k}$ (extended by 0 outside a neighborhood of $x_{0}$ ) defines a global (nonholomorphic) section with the required properties. To make it holomorphic we must correct it with the equation $\bar{\partial} u_{k}=\bar{\partial} g_{k}$ and define $s_{k}=g_{k}-u_{k}$. We need to make sure that the correction $u_{k}$ is globally small.

One technical difficulty arises: the Hörmander estimates for the $\bar{\partial}$-equation deal with $(n, 1)$-forms rather than $(0,1)$-forms. We can always twist the line bundle $L$ with the canonical bundle to shift from $(0,1)$-forms to $(n, 1)$-forms. In this case this is delicate because while twisting the bundle we could lose its positivity since $L$ is only semipositive 
and there is no maneuvering room. For this purpose we will need to change the metric on $L$ to make it strictly positive while preserving the estimates in the original metric. This can be achieved by averaging the original metric $\phi$ on $L$ with the metric $\phi_{s}$ that is singular and strictly positive on $L$. That is the reason we need to work with the more sophisticated Demailly-Nadel estimates on singular metrics rather than the Hörmander estimates. More precisely, let us define a new metric $\widetilde{\phi}_{k}$ on $L^{k}$ as follows:

$$
\widetilde{\phi}_{k}=(k-N) \phi+N \phi_{s}-C,
$$

where $N$ and $C$ are large constants, that do not depend on $k$, to be chosen. This is a well defined singular metric on $L^{k}$ since $\widetilde{\phi}_{k}=k \phi+N\left(\phi_{s}-\phi\right)-C$ and the difference of two metrics $\phi_{s}-\phi$ is a well defined function on $X$.

The bundle $L^{k}$ can be expressed as $L^{k}=K_{X} \otimes F_{k}$, where $K_{X}$ is the canonical line bundle. If we endow $L^{k}$ with the metric $\widetilde{\phi}_{k}$ and $K_{X}$ with the metric inherited from the Hermitian metric on $X$, the curvature of $F_{k}$ is

$$
c\left(F_{k}\right)=c\left(\widetilde{\phi}_{k}\right)-c\left(K_{X}\right)=(k-N) c(\phi)+N c\left(\phi_{S}\right)-c\left(K_{X}\right) \geq N \varepsilon \omega-c\left(K_{X}\right)
$$

if $k>N$, and thus it has positive curvature if we take $N$ large enough, where $c(\cdot)$ denotes the curvature form of the corresponding line bundle or metric specified. In fact on the support of $\bar{\partial} g_{k}$ the curvature satisfies $c\left(F_{k}\right) \gtrsim k \omega$.

The metric $\phi_{s}$ is bounded above because it is in $L_{\text {loc }}^{1}$ and is plurisubharmonic. Thus we can take the constant $C$ large enough in (46) in such a way that $\widetilde{\phi}_{k} \leq k \phi$.

The $L^{2}$ norm of $\bar{\partial} g_{k}$ with the metric $\widetilde{\phi}_{k}$ is comparable to the $L^{2}$ norm with respect to the metric $k \phi$ because $\phi_{s}$ is smooth on the support of $\bar{\partial} g_{k}$, thus its norm is bounded by $k^{1-n} M^{-2}\|f\|^{2}$. If we solve the $\bar{\partial}$ equation using the estimates provided by the DemaillyNadel theorem with data that is an $(n, 1)$-form with values in $F_{k}$, we get a solution $u_{k}$ to $\bar{\partial} u_{k}=\bar{\partial} g_{k}\left(u_{k}\right.$ is a global $(n, 0)$-form with values in $F_{k}$ or equivalently a global section of $L^{k}$ ) with $L^{2}$ size controlled by a constant times ${ }^{-n} M^{-2}\|f\|^{2}$ as desired. A priori the norm control of $u_{k}$ is with respect to $\widetilde{\phi}_{k}$, but as $\widetilde{\phi}_{k} \leq k \phi$ we get the desired result.

We proceed now to prove the sampling part of Theorem 6. Given any function $f$ in the Fock space we take a large $M>0$ so that

$$
\int_{|z|>M}|f|^{2} e^{-|z|^{2}} \leq 0.1\|f\|^{2} .
$$

We can construct a sequence of sections $s_{k}$ such that the conclusions of the approximation lemma hold. For such $s_{k}$ the sampling property of $\Lambda_{k}$ can be applied and we have

$$
\left\|s_{k}\right\|^{2} \lesssim \frac{1}{k^{n}} \sum_{\lambda \in \Lambda_{k}}\left|f_{k}(\lambda)\right|^{2} e^{-k \phi(\lambda)} .
$$

Since all the $f_{k}$ have $L^{2}$ norm very small outside the region parametrized by $|z|<M / \sqrt{k}$, which we denote by $U_{k}$, the mean value property implies that

$$
\left\|s_{k}\right\|^{2} \lesssim \frac{1}{k^{n}} \sum_{\lambda \in \Lambda_{k} \cap U_{k}}\left|f_{k}(\lambda)\right|^{2} e^{-k \phi(\lambda)} .
$$


We recall that $k^{n}\left\|s_{k}\right\|^{2} \simeq\|f\|^{2}$, and taking weak limits of $\Sigma_{k}$ implies that

$$
\|f\|^{2} \lesssim \sum_{|\sigma| \leq M}|f(\sigma)|^{2} e^{-|\sigma|^{2}}
$$

\section{The one-dimensional case}

In this section we return to positive line bundles, and focus on the case when $\operatorname{dim}(X)=1$, i.e. we are dealing with a compact Riemann surface. In this case we have a more precise result, namely a full characterization of the interpolation and sampling arrays given by Theorem 4 above.

9.1. The sampling part of Theorem 4 can be reformulated as follows.

Theorem 8. Let $\Lambda$ be a separated array and let $L$ be a holomorphic line bundle over a compact Riemann surface $X$ endowed with a smooth positive metric $\phi$. Then $\Lambda$ is a sampling array for the line bundle $L$ if and only if there are $\varepsilon, r, k_{0}>0$ such that for all $k \geq k_{0}$,

$$
\frac{\#\left(\Lambda_{k} \cap B(x, r / \sqrt{k})\right)}{\int_{B(x, r / \sqrt{k})} i k \partial \bar{\partial} \phi}>\frac{1}{\pi}+\varepsilon \quad \forall x \in X .
$$

Note that the metric in $X$ used to define the balls in (47) is irrelevant, since the density inequality is invariant under change of metric. We will prove this invariance in an arbitrary dimension. Assume that we have two different metrics that induce two distances $d_{1}$ and $d_{2}$ and two volumes $V_{1}$ and $V_{2}$. Suppose that (47) holds for the first metric. Denote $\mu_{k}:=$ $k^{-n} \sum_{\lambda \in \Lambda_{k}} \delta_{\lambda}$ and $v:=(i \partial \bar{\partial} \phi)^{n}$. The hypothesis (47) (in dimension $n$ ) can be written as

$$
\int_{B_{1}(y, r / \sqrt{k})} d \mu_{k}(x) \geq\left(\frac{1}{\pi^{n} n !}+\varepsilon\right) \int_{B_{1}(y, r / \sqrt{k})} d \nu .
$$

We need some notation to check that (48) is invariant under change of metric. Denote

$$
\begin{aligned}
\tilde{f}_{r}(z) & :=\frac{1}{\lambda_{0}(r / \sqrt{k})} \int_{B_{1}(z, r / \sqrt{k})} f(y) d V_{1}(y) \\
& =\frac{1}{\lambda_{0}(r / \sqrt{k})} \int_{X} f(y) \mathbb{1}_{B_{1}(y, r / \sqrt{k})}(z) d V_{1}(y),
\end{aligned}
$$

where $\lambda_{0}(r)$ denotes as in [Blü90] the volume of a Euclidean ball of radius $r$ in $\mathbb{R}^{2 n}$. Thus

$$
\int_{X} \widetilde{f}_{r} d \mu_{k}=\frac{1}{\lambda_{0}(r / \sqrt{k})} \int_{X} f(y) \mu_{k}\left(B_{1}(y, r / \sqrt{k})\right) d V_{1}(y) .
$$

For any $f \geq 0$, by (48) we have

$$
\int_{X} \tilde{f}_{r} d \mu_{k} \geq\left(\frac{1}{\pi^{n} n !}+\varepsilon\right) \int_{X} \tilde{f}_{r} d \nu
$$

We choose $f:=\mathbb{1}_{B_{2}(x, R / \sqrt{k})}$; then

$$
\mathbb{1}_{B_{2}(x,(R-c r) / \sqrt{k})} \leq f_{r} \leq \mathbb{1}_{B_{2}(x,(R+c r) / \sqrt{k})},
$$


where

$$
f_{r}(z):=\frac{1}{V_{1}\left(B_{1}(z, r / \sqrt{k})\right)} \int_{B_{1}(z, r / \sqrt{k})} f(y) d V_{1}(y) .
$$

The following inequalities are now elementary:

$$
\begin{aligned}
& \mu_{k}\left(B_{2}(x,(R+c r) / \sqrt{k})\right) \geq \int_{X} f_{r} d \mu_{k} \\
& =\int_{X} \widetilde{f}_{r} d \mu_{k}+\int_{X}\left(f_{r}-\widetilde{f}_{r}\right) d \mu_{k} \geq\left(\frac{1}{\pi^{n} n !}+\varepsilon\right) \int_{X} \widetilde{f}_{r} d \nu+\int_{X}\left(f_{r}-\widetilde{f}_{r}\right) d \mu_{k} \\
& =\left(\frac{1}{\pi^{n} n !}+\varepsilon\right) \int_{X} f_{r} d \nu+\left(\frac{1}{\pi^{n} n !}+\varepsilon\right) \int_{X}\left(\tilde{f}_{r}-f_{r}\right) d \nu+\int_{X}\left(f_{r}-\widetilde{f}_{r}\right) d \mu_{k} \\
& \geq\left(\frac{1}{\pi^{n} n !}+\varepsilon\right) v\left(B_{2}(x,(R-c r) / \sqrt{k})\right)+\left(\frac{1}{\pi^{n} n !}+\varepsilon\right) \int_{X}\left(\widetilde{f}_{r}-f_{r}\right) d \nu+\int_{X}\left(f_{r}-\widetilde{f}_{r}\right) d \mu_{k} .
\end{aligned}
$$

We aim to prove that

$$
\mu_{k}\left(B_{2}(x,(R+c r) / \sqrt{k})\right) \geq\left(\frac{1}{\pi^{n} n !}+\frac{\varepsilon}{2}\right) v\left(B_{2}(x,(R+c r) / \sqrt{k})\right) .
$$

Clearly if $R$ is large enough $(R \gg c r)$, then by (37),

$$
v\left(\left\{y: \frac{R-r c}{\sqrt{k}} \leq d_{2}(y, x) \leq \frac{R+c r}{\sqrt{k}}\right\}\right) \leq \frac{\varepsilon}{4} v\left(B_{2}(x,(R-r c) / \sqrt{k})\right) .
$$

We still need to prove that the terms $\left(1 /\left(\pi^{n} n !\right)+\varepsilon\right) \int_{X}\left(\widetilde{f}_{r}-f_{r}\right) d v+\int_{X}\left(f_{r}-\widetilde{f}_{r}\right) d \mu_{k}$ are negligible when compared to $v\left(B_{2}(x,(R-r c) / \sqrt{k})\right) \simeq R^{2 n} / k^{n}$ as $k \rightarrow \infty$.

Observe that $\left|f_{r}-\widetilde{f}_{r}\right| \leq K_{1}(r / \sqrt{k}) f_{r}$, where $K_{1}(s)=\sup _{X}\left|1-V_{1}\left(B_{1}(x, s)\right) / \lambda_{0}(s)\right|$. The distortion function $K_{1}(s)$ is $O\left(s^{2}\right)$ [Blü90, Lemma 2], and thus

$$
\left|\int_{X}\left(f_{r}-\tilde{f}_{r}\right) d \nu\right| \leq K_{1}\left(\frac{r}{\sqrt{k}}\right) \int_{X} f_{r} d \nu \leq K_{1}\left(\frac{r}{\sqrt{k}}\right) v\left(B\left(x, \frac{R+c r}{\sqrt{k}}\right)\right) \lesssim \frac{1}{k^{n+1}} .
$$

We assume that $\Lambda$ is separated, thus $\mu_{k}((x,(R+c r) / \sqrt{k})) \lesssim R^{2 n} / k^{n}$, and therefore

$$
\left|\int_{X}\left(f_{r}-\widetilde{f}_{r}\right) d \mu_{k}\right| \leq K_{1}\left(\frac{r}{\sqrt{k}}\right) \int_{X} f_{r} d \nu \leq K_{1}\left(\frac{r}{\sqrt{k}}\right) \mu_{k}\left(B\left(x, \frac{R+c r}{\sqrt{k}}\right)\right) \lesssim \frac{1}{k^{n+1}},
$$

and if we take $k$ large enough, we have proved (49), and the invariance of the density condition under changes of metric follows.

9.2. We proceed now to the proof of Theorem 8 . We start by proving that under the density hypothesis (47) the array $\Lambda$ is sampling. We will first prove that it is $L^{\infty}$-sampling.

Definition 6. We say that a separated array $\Lambda=\left\{\Lambda_{k}\right\}$ is $L^{\infty}$-sampling if there is $k_{0}$ and a constant $0<C<\infty$ such that, for each $k \geq k_{0}$ and any section $s \in H^{0}\left(L^{k}\right)$,

$$
\sup _{x \in X}|s(x)| \leq C \sup _{\lambda \in \Lambda_{k}}|s(\lambda)| \text {. }
$$


If this were not true then for infinitely many $k$ 's there would be $s_{k} \in H^{0}\left(L^{k}\right)$ and points $x_{k} \in X$ such that

$$
\sup _{X}\left|s_{k}\right|=\left|s_{k}\left(x_{k}\right)\right|=1,
$$

and

$$
\sup _{\lambda \in \Lambda_{k}}\left|s_{k}(\lambda)\right|=o(1) .
$$

We take normal coordinates around $x_{k}$ (see Definition 3), and we consider as before arrays $\Lambda_{k} \subset X$ and the dilated sequences $\Sigma_{k} \subset B\left(0, M_{k}\right)$ in $\mathbb{C}$. Since $\Sigma_{k}$ are separated, there is a subsequence converging weakly to $\Sigma$, which for simplicity we keep denoting by $\Sigma_{k}$. The hypothesis implies that

$$
\frac{\#\left(\Sigma \cap B\left(y, r_{0}\right)\right)}{r_{0}^{2}} \geq \frac{1}{\pi}+\varepsilon,
$$

the balls $B\left(y, r_{0}\right)$ are standard balls in $\mathbb{C}$ because we may choose a metric in $X$ such that when dilating by a factor $1 / \sqrt{k}$ the metric around $x$ transported from $X$ to $\mathbb{C}$ by the normal coordinates it converges to the Euclidean metric in $\mathbb{C}$. By a theorem of Seip and Wallsten [SW92, Theorem 1.1], $\Sigma$ is sampling for the space $\mathcal{B F} \mathcal{F}^{\infty}$ of all entire functions such that $\left.\sup |f| e^{-|z|}\right|^{2}<\infty$. On the other hand we may extract a subsequence of functions $f_{k}$ that represent the sections $s_{k}$ in normal coordinates, and converge to $f \in \mathcal{B F}^{\infty}$ such that $|f(0)|=1$ and $\left.f\right|_{\Sigma}=0$; this contradicts the fact that $\Sigma$ is sampling for $\mathcal{B F}^{\infty}$.

Once we know that $\Lambda$ is $L^{\infty}$-sampling it is possible to argue as with the Fekete points that $\left\{\Lambda_{(1+\varepsilon) k}\right\}$ is $L^{2}$-sampling.

Proposition 1. If $\Lambda=\left\{\Lambda_{k}\right\}$ is $L^{\infty}$-sampling then $\left\{\Lambda_{(1+\varepsilon) k}\right\}$ is $L^{2}$-sampling.

Proof. We know by hypothesis that $\sup _{X}|s| \leq C \sup _{\Lambda_{k}}\left|s\left(\lambda_{k}\right)\right|$ for any $s \in H^{0}\left(L^{k}\right)$. In this case it is elementary to check that $\left\{\Lambda_{(1+\varepsilon) k}\right\}$ is also $L^{\infty}$-sampling. For any $s \in$ $H^{0}\left(L^{k}\right)$ and $y \in X$ we define the section

$$
p_{y}(x)=s(x) \otimes\left[\frac{\Phi_{y}^{(\varepsilon / 2) k}(x)}{\left|\Pi_{(\varepsilon / 2) k}(y, y)\right|}\right]^{2} \in H^{0}\left(L^{(1+\varepsilon) k}\right) .
$$

Let us now take $y \in X$ where $|s|$ attains its maximum. Then

$$
\sup _{X}|s|=|s(y)|=\left|p_{y}(y)\right| \leq C \sup _{\Lambda_{(1+\varepsilon) k}}\left|p_{y}(\lambda)\right| \leq C \sup _{\Lambda_{(1+\varepsilon) k}}|s(\lambda)| .
$$

Moreover for any $z \in X$, since $\Lambda$ is sampling,

$$
\begin{aligned}
|s(z)| & =\left|p_{z}(z)\right| \lesssim \sup _{\Lambda_{(1+\varepsilon) k}}|s(\lambda)|\left|\frac{\Phi_{z}^{(\varepsilon / 2) k}(\lambda)}{\left|\Pi_{(\varepsilon / 2) k}(z, z)\right|}\right|^{2} \leq \sum_{\Lambda_{(1+\varepsilon) k}}|s(\lambda)|\left|\frac{\Phi_{z}^{(\varepsilon / 2) k}(\lambda)}{\left|\Pi_{(\varepsilon / 2) k}(z, z)\right|}\right|^{2} \\
& =\sum_{\Lambda_{(1+\varepsilon) k}}|s(\lambda)|\left|\frac{\Pi_{(\varepsilon / 2) k}(z, \lambda)}{\left|\Pi_{(\varepsilon / 2) k}(z, z)\right|}\right|^{2} .
\end{aligned}
$$


Recall that $\left|\Pi_{(\varepsilon / 2) k}(z, z)\right| \simeq \varepsilon k$. Thus if we integrate both sides, we get

$$
\int_{X}|s(z)| \lesssim \frac{1}{\varepsilon k} \sum_{\Lambda_{(1+\varepsilon) k}}|s(\lambda)|
$$

Interpolating between (50) and (51) we obtain

$$
\int_{X}|s(z)|^{2} \lesssim \frac{1}{\varepsilon k} \sum_{\Lambda_{(1+\varepsilon) k}}|s(\lambda)|^{2},
$$

as stated.

Finally, since the hypothesis of Theorem 8 is an open condition, we can conclude that actually $\left\{\Lambda_{(1-\varepsilon) k}\right\}$ is $L^{\infty}$-sampling and therefore $\Lambda$ is $L^{2}$-sampling.

We turn now to the necessity of the density condition. We assume that $\Lambda$ is a sampling array. We already know by Corollary 1 that the density of $\Lambda$ is greater than or equal to a critical level. We need a strict inequality. We prove now that if $\Lambda$ is a sampling array then there is an $\varepsilon>0$ such that $\left\{\Lambda_{(1-\varepsilon) k}\right\}$ is still an $L^{2}$-sampling array.

We know by Theorem 6 than any weak limit $\Sigma \in W(\Lambda)$ is a sampling sequence in $\mathcal{B F}^{2}(\mathbb{C})$. Thus by the description of sampling sequences for such spaces obtained in [SW92], the lower Beurling density satisfies $D^{-}(\Sigma)>1$. We will prove that under these circumstances there is an $\varepsilon>0$ such that $\left\{\Lambda_{(1-2 \varepsilon) k}\right\}$ is $L^{\infty}$-sampling. Suppose not; then for any $n$ there are sections $s_{k} \in H^{0}\left(L^{k}\right)$ such that $\left\|s_{k}\right\|_{\infty}=1$ and $\left\|\left.s\right|_{\Lambda_{(1-1 / n) k}}\right\|_{\infty}=o(1)$ when $k$ is very large. Fix $n$. By passing to a subsequence in normal coordinates around the points $x_{k}$ where $\left|s_{k}\right|$ takes its maximum value, we can construct $f_{n} \in \mathcal{B F}^{\infty}(\mathbb{C})$ of norm one such that $f_{n}(0)=1$ and $\left.f_{n}\right|_{\Sigma_{n}} \equiv 0$, where $\Sigma_{n}$ is a weak limit of a subsequence of $\Lambda_{(1-1 / n) k}$ as $k \rightarrow \infty$ in normal coordinates scaled appropriately. We take another subsequence of the functions $f_{n}$ and of the separated sequences $\Sigma_{n}$ in such a way that $\Sigma_{n}$ converges weakly to $\Sigma, f_{n} \rightarrow f$ and $f \in \mathcal{B F}^{\infty}(\mathbb{C})$ has norm one, $f(0)=1,\left.f\right|_{\Sigma} \equiv 0$ and $\Sigma \in W(\Lambda)$. This is a contradiction since $D^{-}(\Sigma)>1$.

We have proved that $\left\{\Lambda_{(1-2 \varepsilon) k}\right\}$ is $L^{\infty}$-sampling. We finish the proof by observing that by Proposition 1 this implies that $\left\{\Lambda_{(1-\varepsilon) k}\right\}$ is $L^{2}$-sampling.

9.3. We now provide a characterization for interpolation arrays.

Theorem 9. Let $\Lambda$ be a separated array and let $L$ be a holomorphic line bundle with a smooth positive metric $\phi$ over a compact Riemann surface $X$. Then $\Lambda$ is an interpolation array for the line bundle $L$ if and only if there are $\varepsilon, r, k_{0}>0$ such that for all $k \geq k_{0}$,

$$
\frac{\#\left(\Lambda_{k} \cap B(x, r / \sqrt{k})\right)}{\int_{B(x, r / \sqrt{k})} i k \partial \bar{\partial} \phi}<\frac{1}{\pi}-\varepsilon \quad \forall x \in X,
$$

Note that the density condition (52) is invariant under change of metric, which can be shown in much the same way as above for (47). We will first check that (52) implies that $\Lambda$ is an interpolation array. We start by the following reduction. 
Proposition 2. Let $\Lambda$ be separated. If there is a $C>0$ such that for every $k \geq k_{0}$ and every $\lambda \in \Lambda_{k}$ there is a section $s_{\lambda} \in H^{0}\left(L^{k}\right)$ with

(i) $\left|s_{\lambda}(\lambda)\right|=1$,

(ii) $\sup _{\lambda} \sum_{\lambda^{\prime} \neq \lambda}\left|s_{\lambda}\left(\lambda^{\prime}\right)\right|<1 / 2$,

(iii) $\sup _{\lambda^{\prime}} \sum_{\lambda \neq \lambda^{\prime}}\left|s_{\lambda}\left(\lambda^{\prime}\right)\right|<1 / 2$,

(iv) $\left\|\sum c_{\lambda} s_{\lambda}\right\|_{2}^{2} \leq C k^{-1} \sum\left|c_{\lambda}\right|^{2}$,

then $\Lambda$ is an interpolation array.

Proof. Let $\ell^{2}\left(\Lambda_{k}\right)$ be endowed with the norm $\|v\|^{2}:=k^{-1} \sum_{\Lambda_{k}}\left|v_{\lambda}\right|^{2}$. We consider the following two operators. The first is the restriction operator $R: H^{0}\left(L^{k}\right) \rightarrow \ell^{2}\left(\Lambda_{k}\right)$ defined as $R(s)=\{s(\lambda)\}$. It is bounded from $H^{0}\left(L^{k}\right)$ endowed with the $L^{2}$ norm by the Plancherel-Pólya inequality (Lemma 2 ) since $\Lambda$ is separated and its norm $\|R\|$ depends only on the separation constant of $\Lambda$.

The second operator is $E: \ell^{2}\left(\Lambda_{k}\right) \rightarrow H^{0}\left(L^{k}\right)$ defined as $E\left(\left\{v_{\lambda}\right\}\right)=$ $\sum\left\langle v_{\lambda}, s_{\lambda}(\lambda)\right\rangle s_{\lambda}(x)$. It is clearly bounded by properties (i) and (iv). If we prove that $R E$ : $\ell^{2} \rightarrow \ell^{2}$ is invertible with $\left\|(R E)^{-1}\right\|$ bounded independently of $k$, then clearly $\Lambda$ is an interpolation array, because any values $\left\{v_{\lambda}\right\}$ are attained by the section $s=E(R E)^{-1}\left(\left\{v_{\lambda}\right\}\right)$ with size control.

But conditions (i)-(iii) imply that the operator $R E-\mathrm{Id}: \ell^{2}\left(\Lambda_{k}\right) \rightarrow \ell^{2}\left(\Lambda_{k}\right)$ has norm bounded by $1 / 2$ by Schur's Lemma. Thus $R E$ is invertible.

To finish the proof of the sufficiency of (52) we are going to construct the sections as in Proposition 2. Around any given $\lambda \in \Lambda_{k}$ we can consider normal coordinates. Since by hypothesis the density is small, the corresponding sequence $\Sigma_{k}$ is an interpolation sequence for the $\mathcal{B F}^{2}$ space in $\mathbb{C}$. Actually since the separation constant is uniform and the density is uniform, by a theorem of Seip and Walsten [SW92, Theorem 1.2], the constants of interpolation for all the sequences $\Sigma_{k}$ around any point $\lambda \in \Lambda_{k}$ will be uniformly bounded for $k \geq k_{0}$. Thus we can construct functions $f_{\lambda}^{k}$ such that $\left|f_{\lambda}^{k}(0)\right|=1$, $\left\|f_{\lambda}^{k}\right\| \leq C$ and $f_{\lambda}^{k}(\sigma)=0$ for all $\sigma \in \Sigma_{k} \backslash 0$. Now we can construct a global section $g_{\lambda} \in H^{0}\left(L^{k}\right)$ such that near $\lambda, g_{\lambda}(z)$ is very close to $f_{\lambda}(z)^{k} e_{L}^{k}(z)$, where $e_{L}^{k}(z)$ is the local frame around $\lambda$ used for the normal coordinates.

In order to do this we define $g_{\lambda}=\chi_{\lambda, k}(z) f_{\lambda}^{k}(z) e^{k}(z)+u$, where $\chi_{\lambda, k}$ is a cutoff function around $\lambda$ such that $g_{\lambda}(z)=0$ if $d(z, \lambda)>2 C / \sqrt{k}$ and $g_{\lambda}(z)=1$ if $d(z, \lambda)<C / \sqrt{k}$ and $u$ is the solution to the equation $\bar{\partial} u=\bar{\partial} \chi_{\lambda, k} f_{\lambda}^{k}(z) e^{k}(z)$ provided by the Hörmander theorem. This theorem ensures that $\|u\|^{2} \leq \varepsilon$, provided that the cutoff constant $C$ is large enough.

This is not enough if we want the decay needed in Proposition 2, in particular in items (ii)-(iv). We are again going to use the extra freedom that we have because the hypothesis is an open condition. We could have taken $f_{\lambda}^{k}$ such that $\int\left|f_{\lambda}^{k}\right|^{2} e^{-(1-\varepsilon)|z|^{2}}<\infty$ and in this case we could have constructed $g_{\lambda} \in H^{0}\left(L^{(1-\varepsilon) k}\right)$ such that

$$
\left|g_{\lambda}(\lambda)\right|=1, \quad\left\|g_{\lambda}\right\|^{2} \leq C / k, \quad k^{-1} \sum_{\lambda^{\prime} \neq \lambda}\left|g_{\lambda}\left(\lambda^{\prime}\right)\right|^{2} \leq \varepsilon,
$$

and we can take in the construction $\varepsilon>0$ as small as we want without affecting the $K$. 

that

We define $s_{\lambda}(x)=g_{\lambda}(x) \otimes\left[\Phi_{\lambda}^{\varepsilon k / 2}(x) /\left|\Pi_{(\varepsilon / 2) k}(\lambda, \lambda)\right|\right]^{2}$. Using (9) it is easy to check

$$
\sup _{X}\left|\sum_{\Lambda_{k}} c_{\lambda} s_{\lambda}(x)\right| \lesssim \sup _{\Lambda_{k}}\left|c_{\lambda}\right| \quad \text { and } \quad \int_{X}\left|\sum_{\Lambda_{k}} c_{\lambda} s_{\lambda}(x)\right| \lesssim k^{-1} \sum_{\Lambda_{k}}\left|c_{\lambda}\right| .
$$

Thus by interpolation we get $\left\|\sum c_{\lambda} s_{\lambda}\right\|_{2}^{2} \leq C k^{-1} \sum\left|c_{\lambda}\right|^{2}$, which gives (iv). Finally, (ii) and (iii) can be checked in a similar way.

9.4. We turn now to the neccesity of the density condition (52). We need to check that the density condition that we proved to be necessary in Corollary 1 is actually a strict density condition. As a technical tool to prove the necessity of the strict inequality we need to work with $L^{1}$-interpolation arrays. The definition is the following:

Definition 7. We say that a separated array $\Lambda=\left\{\Lambda_{k}\right\}$ is an $L^{1}$-interpolation array if there is $k_{0}$ and a constant $0<C<\infty$ such that for each $k \geq k_{0}$ and any set of vectors $\left\{v_{\lambda}\right\}_{\lambda \in \Lambda_{k}}$ (each $v_{\lambda}$ is an element of the fiber of $\lambda$ in $L^{k}$ ) there is a section $s \in H^{0}\left(L^{k}\right)$ such that

$$
s(\lambda)=v_{\lambda}, \quad \lambda \in \Lambda_{k}
$$

and

$$
\int_{X}|s(x)| \leq C k^{-1} \sum_{\lambda \in \Lambda_{k}}\left|v_{\lambda}\right|
$$

At each level $k \geq k_{0}$, the best constant $C_{k}$ such that (53) holds for all $s \in H^{0}\left(L^{k}\right)$ that interpolate the prescribed values, is called the constant of interpolation at level $k$. Of course $\Lambda$ is an interpolation array if all the constants $\left\{C_{k}\right\}$ are uniformly bounded. There is an alternative way of computing $C_{k}$ by duality.

Proposition 3. The constant of $L^{1}$-interpolation at level $k$ is comparable to the smallest constant $A_{k}$ such that

$$
\sup _{x \in X} k^{-1}\left|\sum_{\Lambda_{k}}\left\langle a_{\lambda}, \Pi_{k}(x, \lambda)\right\rangle\right| \leq A_{k} \sup _{\Lambda_{k}}\left|a_{\lambda}\right|,
$$

where $\left\{a_{\lambda}\right\}_{\lambda \in \Lambda_{k}}$ are arbitrary elements in the fiber of $\lambda$ in $L^{k}$.

Proof. This is standard and follows from the fact that the Bergman kernel decays very fast away from the diagonal (9). Thus the Bergman projection from the sections of $L^{k}$ endowed with the $L^{p}$ norm to the holomorphic sections endowed with the $L^{p}$ norm is bounded for all $p \in[1, \infty]$, and the dual space of $H^{0}\left(L^{k}\right)$ with the $L^{1}$ norm is the space $H^{0}\left(L^{k}\right)$ endowed with the supremum norm.

It will be convenient to compare interpolation arrays in $L^{1}$ and in $L^{2}$ and we will use the following proposition.

Proposition 4. If $\Lambda=\left\{\Lambda_{k}\right\}$ is an $L^{1}$-interpolation array then $\left\{\Lambda_{(1-\varepsilon) k}\right\}$ is an $L^{2}$ interpolation array. 
Proof. If $\Lambda$ is an $L^{1}$-interpolation array then for each $\lambda \in \Lambda_{(1-2 \varepsilon) k}$ we can build a "Lagrange type" section $s_{\lambda} \in H^{0}\left(L^{(1-2 \varepsilon) k}\right)$ such that $\left|s_{\lambda}(\lambda)\right|=1,\left|s_{\lambda}\left(\lambda^{\prime}\right)\right|=0$ for all $\lambda^{\prime} \in \Lambda_{(1-2 \varepsilon) k} \backslash\{\lambda\}$, and $\left\|s_{\lambda}\right\|_{L^{1}} \leq C / k$. Then by the sub-mean value property (10) we obtain $\sup _{X}\left|s_{\lambda}(x)\right| \leq C k\left\|s_{\lambda}\right\|_{L^{1}} \leq C$. Thus we can use the same argument as in Theorem 6 to prove that $\left\{\Lambda_{(1-\varepsilon) k}\right\}$ is an $L^{2}$-interpolation array.

The proof of the strict inequality (52) follows once we establish the following:

Proposition 5. Assume that $\operatorname{dim}(X)=1$. Let $\Lambda$ be an $L^{2}$-interpolation array. There is an $\varepsilon>0$ such that $\left\{\Lambda_{(1+\varepsilon) k}\right\}$ is an $L^{2}$-interpolation array.

Proof. We know by Theorem 6 than any weak limit $\Sigma \in W(\Lambda)$ is an interpolating sequence in $\mathcal{B F}^{2}(\mathbb{C})$. Thus by the description of interpolating sequences for such spaces obtained in [Sei92], the upper Beurling density satisfies $D^{+}(\Sigma)<1$. We will prove that under these circumstances there is an $\varepsilon>0$ such that $\left\{\Lambda_{(1+2 \varepsilon) k}\right\}$ is an $L^{1}$-interpolation array.

Suppose not; then, for any $n$ the interpolation constants $C_{k}$ at level $k$ for $\Lambda_{(1+1 / n) k}$ blow up. Thus by the dual description of $C_{k}$ given in Proposition 3 we can find sequences $\left\{a_{\lambda}\right\}_{\lambda \in \Lambda_{(1+1 / n) k}}$ of vectors such that $\sup _{\Lambda_{(1+1 / n) k}}\left|a_{\lambda}\right|=1$ and

$$
\sup _{x \in X} k^{-1}\left|\sum_{\Lambda(1+1 / n) k}\left\langle a_{\lambda}, \Pi_{k}(x, \lambda)\right\rangle\right|=o(1) \quad \text { as } k \rightarrow \infty .
$$

Fix $n$. By passing to a subsequence in normal coordinates around the points $\lambda_{k}^{*}$ where $\left|a_{\lambda}\right|$ takes its maximum value, we can extract a subsequence of $\Lambda_{(1+1 / n) k}$ in normal coordinates that scaled appropriately converges weakly as $k \rightarrow \infty$ to the separated sequence $\Sigma_{n} \subset \mathbb{C}$. Moreover, after taking a subsequence again, there are subsequences $a_{\lambda}^{k} \rightarrow a_{\sigma}^{n}$ for all $\sigma \in \Sigma_{n}$. We are going to prove that in this case

$$
f_{n}(z):=\sum_{\sigma \in \Sigma_{n}} a_{\sigma}^{n} e^{\bar{\sigma} z-1 / 2|\sigma|^{2}} \equiv 0
$$

with $\left|a_{0}\right|=1$ and $\sup _{\sigma}\left|a_{\sigma}^{n}\right| \leq 1$.

To see this we will show that $\sup _{|z|<1}\left|f_{n}(z)\right| e^{-|z|^{2}} \leq \varepsilon$ for any $\varepsilon>0$.

Observe that since $\Sigma_{n}$ is separated and $\left|a_{\sigma}^{n}\right| \leq 1$, the decay of the Bargmann-Fock kernel away from the diagonal implies that for any $\varepsilon>0$ one can find $R>0$ such that

$$
\sup _{|z|<1}\left|\sum_{\sigma^{n} \in \Sigma_{n},|\sigma|>R} a_{\sigma}^{n} e^{\bar{\sigma} z-\frac{1}{2}|\sigma|^{2}}\right| e^{-\frac{1}{2}|z|^{2}} \leq \varepsilon .
$$

So we only need to care about the points $\sigma \in \Sigma_{n} \cap B(0, R)$. But these can be dealt with because, with certain abuse of notation,

$$
k^{-1} \sum_{\lambda \in \Lambda_{(1+1 / n) k} \cap B\left(\lambda_{k}^{*}, R / \sqrt{k}\right)}\left\langle a_{\lambda}, \Pi_{k}(x, \lambda)\right\rangle \rightarrow \frac{1}{\pi} \sum_{\sigma \in \Sigma_{n},|\sigma|<R} a_{\sigma}^{n} e^{\bar{\sigma} z-\frac{1}{2}|\sigma|^{2}-\frac{1}{2}|z|^{2}}
$$

uniformly in $|z|<1$ when the section is expressed in appropriately scaled normalized coordinates around $\lambda_{k}^{*}$. This property is usually called the universality of the reproducing kernels and it is proved in [BSZ00, Theorem 3.1]. Actually in [BSZ00] it is assumed 
that $X$ is equipped with the metric induced by the curvature of the line bundle, but since condition (52) is invariant under change of metric, we may also assume that this is the case. We have

$$
k^{-1}\left|\sum_{\lambda \in \Lambda_{(1+1 / n) k} \cap B\left(\lambda_{k}^{*}, R / \sqrt{k}\right)}\left\langle a_{\lambda}, \Pi_{k}(x, \lambda)\right\rangle\right| \leq \varepsilon
$$

for $k$ large enough because the global sum for all $\lambda \in \Lambda_{(1+1 / n) k}$ converges to zero and the terms $\lambda$ outside the ball $B\left(\lambda_{k}^{*}, R / \sqrt{k}\right)$ are small when $R$ is large because $\Lambda_{(1+1 / n) k}$ is separated and there is a fast decay of the normalized reproducing kernel away from the diagonal (9).

Finally we have proved that $f_{n} \equiv 0$ and $\left\{a_{\sigma}^{n}\right\}$ is a uniformly bounded sequence with $a_{0}=1$. We can take a subsequence as $n \rightarrow \infty$ and we find that $\Sigma_{n} \rightarrow \Sigma$ weakly and there is a bounded sequence $\left\{a_{\sigma}\right\}$ such that $f(z)=\sum a_{\sigma} e^{\bar{\sigma} z-|\sigma|^{2} / 2} \equiv 0$ and $\left|a_{0}\right|=1$. This is clearly not possible since $\Sigma \in W(\Lambda)$ and so $D^{+}(\Lambda)<1$, thus $\Lambda$ is interpolating for the $L^{1}$ Bargmann-Fock space and this means that by duality

$$
\sup _{\sigma}\left|a_{\sigma}\right| \leq C \sup _{z \in \mathbb{C}}\left|\sum a_{\sigma} e^{\bar{\sigma} z-|\sigma|^{2} / 2}\right| e^{-|z|^{2}}
$$

We have thus proved that $\left\{\Lambda_{(1+2 \varepsilon) k}\right\}$ is an $L^{1}$-interpolation array. By Proposition 4 this implies that $\left\{\Lambda_{(1+\varepsilon) k}\right\}$ is an $L^{2}$-interpolation array.

Acknowledgments. Part of this work was done while Nir Lev was staying at the Centre de Recerca Matemàtica (CRM) in Barcelona, and he would like to express his gratitude to the institute for hospitality and support during his stay.

Nir Lev is partially supported by the Israel Science Foundation grant No. 225/13 and Joaquim Ortega-Cerdà is supported by the project MTM2014-51834-P and the CIRIT grant 2014SGR-289.

\section{References}

[AOC12] Ameur, Y., Ortega-Cerdà, J.: Beurling-Landau densities of weighted Fekete sets and correlation kernel estimates. J. Funct. Anal. 263, 1825-1861 (2012) Zbl 1256.31001 MR 2956927

[AFK14] Ascensi, G., Feichtinger, H., Kaiblinger, N.: Dilation of the Weyl symbol and BalianLow theorem. Trans. Amer. Math. Soc. 366, 3865-3880 (2014) Zbl 1298.47059 MR 3192621

[BBS08] Berman, R., Berndtsson, B., Sjöstrand, J.: A direct approach to Bergman kernel asymptotics for positive line bundles. Ark. Mat. 46, 197-217 (2008) Zbl 1161.32001 MR 2430724

[BBWN11] Berman, R., Boucksom, S., Witt Nyström, D.: Fekete points and convergence towards equilibrium measures on complex manifolds. Acta Math. 207, 1-27 (2011) Zbl 1241.32030 MR 2863909

[Ber03] Berndtsson, B.: Bergman kernels related to Hermitian line bundles over compact complex manifolds. In: Explorations in Complex and Riemannian Geometry, Contemp. Math. 332, Amer. Math. Soc., Providence, RI, 1-17 (2003) Zbl 1038.32003 MR 2016088

[Ber10] Berndtsson, B.: An introduction to things $\bar{\partial}$. In: Analytic and Algebraic Geometry, IAS/Park City Math. Ser. 17, Amer. Math. Soc., Providence, RI, 7-76 (2010) Zbl 1227.32039 MR 2743815 
[BSZ00] Bleher, P., Shiffman, B., Zelditch, S.: Universality and scaling of correlations between zeros on complex manifolds. Invent. Math. 142, 351-395 (2000) Zbl 0964.60096 MR 1794066

[Blü90] Blümlinger, M.: Asymptotic distribution and weak convergence on compact Riemannian manifolds. Monatsh. Math. 110, 177-188 (1990) Zbl 0717.28001 MR 1084310

[GM13] Grochenig, K., Malinnikova, E.: Phase space localization of Riesz bases for $L^{2}\left(\mathbb{R}^{n}\right)$. Rev. Mat. Iberoamer. 29, 115-134 (2013) Zbl 1262.81081 MR 3010124

[HM11] Hsiao, C. Y., Marinescu, G.: Asymptotics of spectral function of lower energy forms and Bergman kernel of semi-positive and big line bundles. Comm. Anal. Geom. 22, 1-108 (2014) Zbl 1316.32013 MR 3194375

[Kod54] Kodaira, K.: On Kähler varieties of restricted type (an intrinsic characterization of algebraic varieties). Ann. of Math. (2) 60, 28-48 (1954) Zbl 0057.14102 MR 0068871

[Lan67] Landau, H. J.: Necessary density conditions for sampling and interpolation of certain entire functions. Acta Math. 117, 37-52 (1967) Z Zbl 0154.15301 MR 0222554

[Lin01] Lindholm, N.: Sampling in weighted $L^{p}$ spaces of entire functions in $\mathbb{C}^{n}$ and estimates of the Bergman kernel. J. Funct. Anal. 182, 390-426 (2001) Zbl 1013.32008 MR 1828799

[MM07] Ma, X., Marinescu, G.: Holomorphic Morse Inequalities and Bergman Kernels. Progr. Math. 254, Birkhäuser, Basel (2007) Zbl 1135.32001 MR 2339952

[Mar07] Marzo, J.: Marcinkiewicz-Zygmund inequalities and interpolation by spherical harmonics. J. Funct. Anal. 250, 559-587 (2007) Zbl 1141.43011 MR 2352491

[MOC10] Marzo, J., Ortega-Cerdà, J.: Equidistribution of Fekete points on the sphere. Constr. Approx. 32, 513-521 (2010) Zbl 1203.41003 MR 2726443

[Moř66] Moǔšezon, B. G.: On $n$-dimensional compact complex manifolds having $n$ algebraically independent meromorphic functions. I-III. Izv. Akad. Nauk SSSR Ser. Mat. 30, 133-174, 345-386, 621-656 (1966) (in Russian) Zbl 0161.17802 MR 0216522(I) MR 0216523(II) MR 0216524(III)

[NO12] Nitzan, S., Olevskii, A.: Revisiting Landau's density theorems for Paley-Wiener spaces. C. R. Math. Acad. Sci. Paris 350, 509-512 (2012) Zbl 1248.41010 MR 2929058

[OCP12] Ortega-Cerdà, J., Pridhnani, B.: Beurling-Landau's density on compact manifolds. J. Funct. Anal. 263, 2102-2140 (2012) Zbl 1277.58015 MR 2956935

[RS15] Rota Nodari, S., Serfaty, S.: Renormalized energy equidistribution and local charge balance in 2D Coulomb systems. Int. Math. Res. Notices 2015, 3035-3093. Zbl 1321.82029 MR 3373044

[Sei92] Seip, K.: Density theorems for sampling and interpolation in the Bargmann-Fock space. I. J. Reine Angew. Math. 429, 91-106 (1992) Zbl 0745.46034 MR 1173117

[SW92] Seip, K., Wallstén, R.: Density theorems for sampling and interpolation in the Bargmann-Fock space. II. J. Reine Angew. Math. 429, 107-113 (1992) Zbl 0745.46033 MR 1173118

[Siu84] Siu, Y. T.: A vanishing theorem for semipositive line bundles over non-Kähler manifolds. J. Differential Geom. 19, 431-452 (1984) Zbl 0577.32031 MR 0755233

[Tia90] Tian, G.: On a set of polarized Kähler metrics on algebraic manifolds. J. Differential Geom. 32, 99-130 (1990) Zbl 0706.53036 MR 1064867

[Vil09] Villani, C.: Optimal Transport, Old and New. Grundlehren Math. Wiss. 338, Springer (2009) Zbl 1156.53003 MR 2459454

[Ze198] Zelditch, S.: Szegô kernels and a theorem of Tian. Int. Math. Res. Notices 1998, 317 331 Zbl 0922.58082 MR 1616718 